\title{
GEOMETRIC HEAT COMPARISON CRITERIA FOR RIEMANNIAN MANIFOLDS
}

\author{
LEON KARP AND NORBERT PEYERIMHOFF
}

\begin{abstract}
The main results of this article are small time heat comparison results for two points in two manifolds with characteristic functions as initial temperature distributions (Theorems 1 and 2). These results are based on the geometric concepts of (essential) distance from the complement and spherical area function. We also discuss some other geometric results about the heat development and illustrate them by examples.
\end{abstract}

\section{Introduction: ExAmples AND STATEMENT OF RESUlts}

This article is mainly concerned with small time properties of the heat flow on Riemannian manifolds. Of particular interest are geometric heat comparison criteria for two different points in two manifolds. All Riemannian manifolds $M$ considered in this Introduction are connected, complete and without boundary. We also assume that they have a lower (not necessarily positive) bound on the Ricci curvature, an upper bound on the sectional curvature and a positive lower bound on the injectivity radius. For any closed subset $\Omega \subset M$ with $\operatorname{vol}_{n}(\partial \Omega)=0$ (where $n=\operatorname{dim} M$ and $\operatorname{vol}_{n}$ denotes the Riemannian measure on $\left.M\right)$ let $f_{\Omega, X}:(0, \infty) \times M \rightarrow \mathbb{R}$ denote the smooth solution of the heat equation

$$
\frac{\partial}{\partial t} f(t, x)=\Delta f(t, x), \quad \lim _{t \rightarrow 0^{+}} f(t, x)=\chi_{\Omega}(x) \quad \text { for all } x \in M \backslash \partial \Omega,
$$

where $\chi_{\Omega}$ is the characteristic function of $\Omega$. The solution $f_{\Omega, M}$ is given by

$$
f_{\Omega, M}(t, x)=\int_{\Omega} k_{M}(t, x, y) d y,
$$

where $k_{M}$ is the heat kernel on $M . f_{\Omega, M}(t, \cdot)$ describes the temperature distribution of the heat flow at time $t>0$ for the given initial temperature distribution $\chi_{\Omega}$. Our comparison data are given by triples $(x, \Omega, M)$, where $M$ is a Riemannian manifold (with the above properties), $x \in M$ and $\Omega$ is a closed subset of $M$ with $\operatorname{vol}_{n}(\partial \Omega)=0$. A closed subset $\Omega \subset M$ with $\operatorname{vol}_{n}(\partial \Omega)=0$ is called henceforth admissible.

Definition 1.1. We say that $\left(x_{1}, \Omega_{1}, M_{1}\right)$ is initially hotter than $\left(x_{2}, \Omega_{2}, M_{2}\right)$ if there exists a time $\tau>0$ such that

$$
f_{\Omega_{1}, M_{1}}\left(t, x_{1}\right) \geq f_{\Omega_{2}, M_{2}}\left(t, x_{2}\right) \quad \text { for all } t \in(0, \tau) .
$$

$\left(x_{1}, \Omega_{1}, M_{1}\right)$ is initially strictly hotter than $\left(x_{2}, \Omega_{2}, M_{2}\right)$, if inequality (2) holds strictly.

Date: May 26, 2005.

Mathematics Subject Classification (2000): 58J35, 35K05 
If $\left(x_{1}, \Omega_{1}, M_{1}\right)$ is initially hotter, resp., initially strictly hotter than $\left(x_{2}, \Omega_{2}, M_{2}\right)$, we write shortly

$$
\left(x_{1}, \Omega_{1}, M_{1}\right) \succeq\left(x_{2}, \Omega_{2}, M_{2}\right) \text {, respectively, }\left(x_{1}, \Omega_{1}, M_{1}\right) \succ\left(x_{2}, \Omega_{2}, M_{1}\right) .
$$

We also want to compare the initial temperatures of subsets of two manifolds.

Definition 1.2. We say that the set $I_{1} \subset M_{1}$ is uniformly initially strictly hotter than $I_{2} \subset M_{2}$, if for all $x_{1} \in I_{1}$ and $x_{2} \in I_{2}$ inequality (2) holds strictly with a uniform $\tau>0$. In this case we write $\left(I_{1}, \Omega_{1}, M_{1}\right) \succ\left(I_{2}, \Omega_{2}, M_{1}\right)$.

To state our first result, we need some preparations. Let $\Omega \subset M$ be an admissible subset. Let $B_{r}(x)$ denote the closed ball of radius $r$ about $x$. The function $R$ : $M \rightarrow[0, \infty]$, defined by

$$
R(x):=\sup \left\{r \geq 0 \mid \operatorname{vol}_{n}\left(B_{r}(x)\right)=\operatorname{vol}_{n}\left(B_{r}(x) \cap \Omega\right)\right\},
$$

is called the (essential) distance of $x$ from the complement $\Omega^{c}=M \backslash \Omega$. Note that $x \in \overline{\Omega^{c}}$ implies $R(x)=0$. Moreover, $R(y) \geq R(x)-d(x, y)$ implies that $R$ is continuos. If $\Omega$ is a closed set with piecewise smooth boundary then we have

$$
R(x)=d\left(x, \overline{\Omega^{c}}\right) .
$$

The supremum

$$
R_{\infty}(\Omega):=\sup _{x \in \Omega} R(x)
$$

is called the inradius of the set $\Omega$ and the set

$$
I_{\infty}(\Omega):=\left\{x \in \Omega \mid R(x)=R_{\infty}(\Omega)\right\}
$$

denotes the set of maximally interior points of $\Omega$. Continuity of $R: M \rightarrow[0, \infty]$ implies that $I_{\infty}(\Omega)$ is a closed set.

Let $\operatorname{inj}(x)$ denote the injectivity radius of $M$ at $x$. The distance from the complement plays an important role in the following comparison criterion.

Theorem 1. Let $\left(x_{1}, \Omega_{1}, M_{1}\right)$ and $\left(x_{2}, \Omega_{2}, M_{2}\right)$ be given and let $R_{j}: M_{j} \rightarrow[0, \infty]$ be the corresponding distances from the complements. If $R_{j}\left(x_{j}\right)<\operatorname{inj}\left(x_{j}\right)$, for $j=1,2$, and

$$
R_{1}\left(x_{1}\right)<R_{2}\left(x_{2}\right),
$$

then $\left(x_{2}, \Omega_{2}, M_{2}\right)$ is initially strictly hotter than $\left(x_{1}, \Omega_{1}, M_{1}\right)$.

More general, given $0 \leq R_{1}<R_{2}$ and two compact sets $I_{1} \subset M_{1}$ and $I_{2} \subset M_{2}$ satisfying

(a) $R_{1}\left(x_{1}\right) \leq R_{1}$ for all $x_{1} \in I_{1}$ and $R_{2}\left(x_{2}\right) \geq R_{2}$ for all $x_{2} \in I_{2}$,

(b) $R_{2}<\operatorname{inj}(x)$ for all $x \in I_{1} \cup I_{2}$.

Then $I_{1}$ is uniformly initially strictly hotter than $I_{2}$.

Note that if $\Omega \subset M$ is an admissible set then so is $\overline{\Omega^{c}}$. Using this fact and heat conservation (see property (HK2) in Section 2), Theorem 1 can also be used to compare points outside the domains $\Omega_{j}$.

Corollary 1.3. Let $\left(x_{1}, \Omega_{1}, M_{1}\right)$ and $\left(x_{2}, \Omega_{2}, M_{2}\right)$ be given and

$$
R_{j}^{-}(x):=\sup \left\{r \geq 0 \mid \operatorname{vol}_{n}\left(B_{r}(x) \cap \Omega_{j}\right)=0\right\} \quad \text { for } x \in M_{j} .
$$

If $R_{j}^{-}\left(x_{j}\right)<\operatorname{inj}\left(x_{j}\right)$, for $j=1,2$, and

$$
R_{1}^{-}\left(x_{1}\right)>R_{2}^{-}\left(x_{2}\right),
$$

then $\left(x_{2}, \Omega_{2}, M_{2}\right)$ is initially strictly hotter than $\left(x_{1}, \Omega_{1}, M_{1}\right)$. 
The following example is an easy application of the theorem.

Example 1: Let $\Pi_{1} \subset \mathbb{R}^{2}$ and $\Pi_{2} \subset \mathbb{H}^{2}$ be two regular $n$-gons incribed in a Euclidean and a hyperbolic circle of the same radius $R>0$. Let $x_{1} \in \Pi_{1}$ and $x_{2} \in \Pi_{2}$ be the corresponding centers. Then the radii $r_{1}$ and $r_{2}$ of the corresponding inballs are given by

$$
r_{1}=\cos (\pi / n) R \text { and } \tanh r_{2}=\cos (\pi / n) \tanh R,
$$

and strict concavity of $r \mapsto \tanh (r)$ on $[0, \infty)$ implies that $r_{1}>r_{2}$. Hence, $\left(x_{1}, \Pi_{1}, \mathbb{R}^{2}\right)$ is initially strictly hotter than $\left(x_{2}, \Pi_{2}, \mathbb{H}^{2}\right)$.

Another consequence of Theorem 1 is the existence of a unique initially hottest point if there is a unique point $x \in \Omega$ with largest distance to the boundary (see Corollary 1.5 below). Initially hottest points are defined as follows:

Definition 1.4. Let $\Omega \subset M$ be an admissible subset. $x \in M$ is an initially hottest point of $\Omega$ if and only if

$$
(x, \Omega, M) \succeq\left(x^{\prime}, \Omega, M\right) \quad \text { for all } x^{\prime} \in M .
$$

Corollary 1.5. Let $\Omega \subset M$ be an admissible subset and $R: M \rightarrow[0, \infty]$ be the corresponding distance from the complement. We assume that $R(x)<\operatorname{inj}(x)$ for all $x \in \Omega$. If the set $I_{\infty}(\Omega)$ of maximally interior points consists of only one point then this point is also a unique initially hottest point of $\Omega$.

Corollary [1.5] applies, e.g., to strictly convex compact subsets $\Omega$ of $\mathbb{R}^{n}$.

In ChK-90, Chavel and Karp study the behaviour of the set of hottest points

$$
H(t)=H_{\Omega}(t)=:\left\{x_{0} \in M \mid f_{\Omega, M}\left(t, x_{0}\right)=\max _{x \in M} f_{\Omega, M}(t, x)\right\},
$$

as $t \rightarrow \infty$. The following result gives informations about the set $H(t)$, as $t \rightarrow 0$.

Corollary 1.6. Let $\Omega \subset M$ be a compact admissible set of positive volume, let $R: M \rightarrow[0, \infty)$ be the corresponding distance from the complement, and $H(t)$ be the set of hottest points. We assume that $R_{\infty}(\Omega)<\operatorname{inj}(x)$ for all $x \in \Omega$. Then we have, for any sequence $\left\{x_{j}\right\}$ with $x_{j} \in H\left(t_{j}\right)$, and $t_{j} \rightarrow 0$ :

$$
d\left(x_{j}, I_{\infty}(\Omega)\right) \rightarrow 0
$$

as $j \rightarrow \infty$.

Corollaries 1.5 and 1.6 are used in our next example.

Example 2: Let $\Delta \subset \mathbb{R}^{2}$ be an arbitrary Euclidean triangle. The center of the inball of $\Delta$ is the unique initially hottest point, by Corollary [1.5. The results in ChK-90 imply that the set of hottest points $H(t)$ remains in the triangle $\Delta$ for all $t>0$; moreover, $H(t)$ converges to the center of mass of $\Delta$, as $t \rightarrow \infty$. In combination with Corollary [1.6 we conclude that the map $t \mapsto H(t)$ evolves from the center of the inball and, finally, collapses into the center of mass of the triangle. The precise trajectory of this map is not clear to us. Numerical analysis shows that the trajectory stays close to (but not on) the straight Euclidean arc connecting these two centers. The experiments seem also to indicate that $H(t)$ is always a single point, but we lack a proof of this assumption. However, the next proposition implies that $H(t)$ is a single point at least for sufficiently large $t$. 

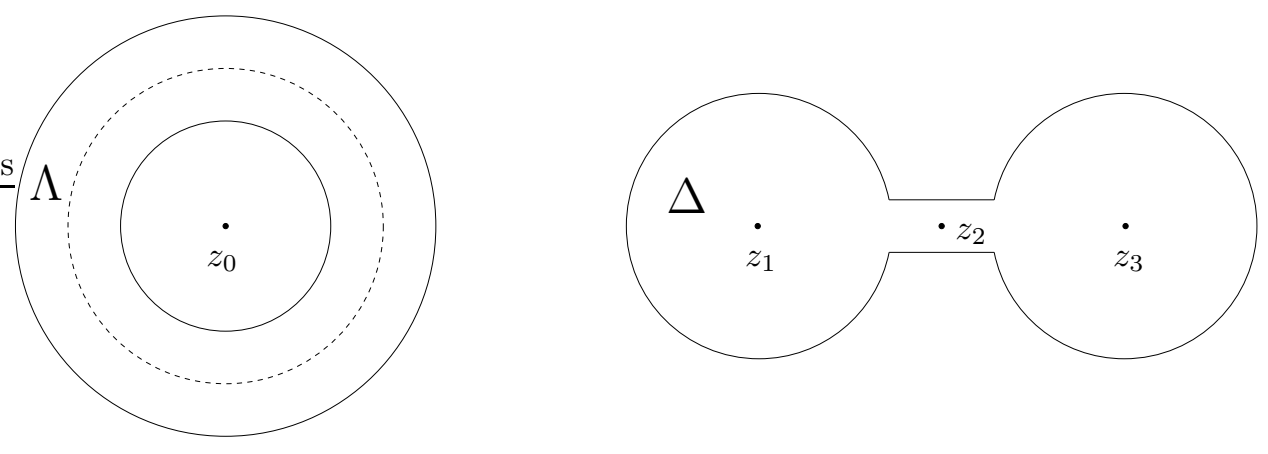

FiguRE 1. Examples 3 and 4: Annulus and dumbbell in $\mathbb{R}^{2}$

Proposition 1.7. Let $\Omega \subset \mathbb{R}^{n}$ be a compact admissibe set of positive volume. Then there is a $T=T(\Omega)>0$ such that $H(t)$ consists of a single point, for all $t \geq T$. Moreover, the map $t \mapsto H(t)$ is a smooth curve and the temperature $t \mapsto f_{\Omega, \mathbb{R}^{n}}(t, H(t))$ at the hottest point is strictly decreasing on the interval $(T, \infty)$.

The monotonicity statement of Proposition 1.7 and the proof of it has been pointed out to us by Oliver Stein. Further applications of Proposition [1.7] are discussed in the next two examples.

Examples 3 and 4: Let $\Lambda$ and $\Delta$ be an Euclidean annulus and a dumbbell, as presented in Figure 1

The initially hottest points of the annulus $\Lambda$ form the dashed circle. By symmetry reasons, the set $H(t)$ of hottest points is spherically symmetric with respect to $z_{0}$, for all $t>0$. By ChK-90, $H(t)$ shrinks to $z_{0}$, as $t \rightarrow \infty$. Now, Proposition 1.7 implies that $H(t)$ arrives at $z_{0}$ in finite time.

$z_{1}$ and $z_{3}$ are the initially hottest points of the dumbbell $\Delta$. Assume that the coordinates of $z_{1}$ and $z_{3}$ are $(-a, 0)$ and $(a, 0)$. An easy argument, using the form of the heat kernel along the vertical axes shows for any point $z=(x, y), y \neq 0$ that the point $(x, 0)$ is initally strictly hotter than $z$. This implies that $H(t) \subset \mathbb{R} \times\{0\}$, for all times $t>0$. By the same reasoning as above we conclude that there is a finite time $T>0$ such that $H(t)=\left\{z_{2}\right\}$ for all $t>T$. The development of hottest points of a more simple dumbbell (with square ends) is explicitely discussed in Appendix A

Note that there is no analogue of Proposition [1.7 in hyperbolic space. It was pointed out in ChK-90 that, for $\Omega$ equals a large enough hyperbolic dumbbell, the set $H(t)$ does not converge to a single limit point, as $t \rightarrow \infty$.

Our next result refers to Euclidean and hyperbolic polygons.

Proposition 1.8. Let $M$ be the Euclidean or the hyperbolic plane and $\Sigma_{1}, \Sigma_{2} \subset M$ be two polygons of the same area and the same number of sides. Assume that $\Sigma_{1}$ is regular and that $x_{1}$ is its center. Then we have

$$
f_{\Sigma_{1}, M}\left(t, x_{1}\right) \geq \max _{x \in M} f_{\Sigma_{2}, M}(t, x) .
$$

If (3) holds with equality for some time $t>0$ then $\Sigma_{2}$ is also regular. In particular, we have $\left(x_{1}, \Sigma_{1}, M\right) \succeq\left(x, \Sigma_{2}, M\right)$, for all $x \in M$. 
Now, we move on to examples in which Theorem 1 is not applicable.

Example 5: In Figure 2 the inradius at the point $z_{1}$ in $\Omega_{1}$ coincides with the inradii at the points $z_{2}$ and $z_{3}$ in $\Omega_{2}$. Therefore, the initial heat of $z_{1}$ and $z_{2}$ cannot be compared with the help of Theorem 1
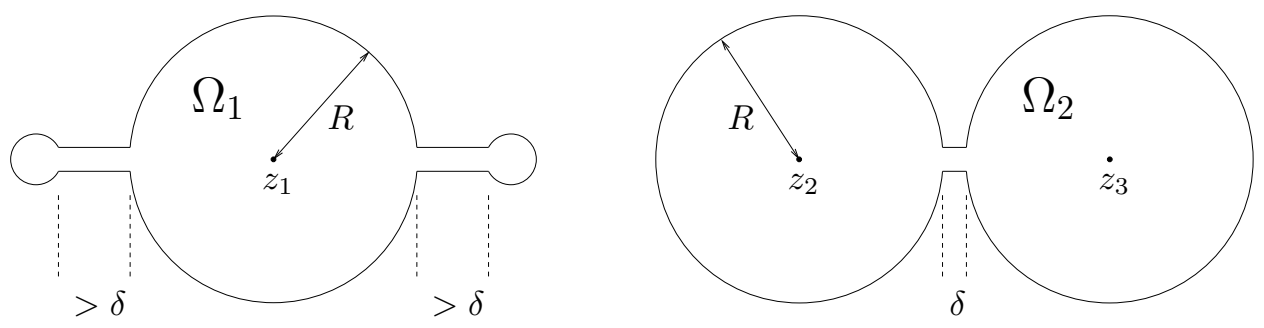

Figure 2. Example 5: Comparison of $\left(z_{1}, \Omega_{1}, \mathbb{R}^{2}\right)$ and $\left(z_{2}, \Omega_{2}, \mathbb{R}^{2}\right)$

Example 6: Let $C \subset \mathbb{R}^{2}$ be a plane curve with absolute curvature bounded from above by a positive constant $k=1 / r>0$. Let $\Sigma \subset \mathbb{R}^{2}$ denote the closed $R$-tube about $C$ of width $R<r$ (see Figure 3). Then Theorem 1 cannot be applied to a pair of points $z_{1}, z_{2}$ on $C$.

To treat the last two examples we introduce a finer criterion which is specially adapted to the cases $M=\mathbb{R}^{n}$ and $M=\mathbb{H}^{n}$. To do so, we first introduce the spherical area function $A:[0, \infty) \rightarrow[0, \infty)$ of a triple $(x, \Omega, M)$ as

$$
A(r):=\operatorname{vol}_{n-1}\left(S_{r}(x) \cap \Omega\right)
$$

where $S_{r}(x)$ denotes the sphere of radius $r$ about $x$.

Theorem 2. Let $M=\mathbb{R}^{n}$ or $M=\mathbb{H}^{n}$ and $\Omega_{1}, \Omega_{2} \subset M$ be two admissible subsets. Let $x_{1}, x_{2} \in M$ and $A_{j}:[0, \infty) \rightarrow[0, \infty)$ be the corresponding spherical area functions. If there exist $0<R<\tilde{R}$ such that the following inequalities are satisfied:

$$
\begin{aligned}
& A_{1}(r) \leq A_{2}(r) \quad \text { for all } r \in(0, R], \\
& A_{1}(r)<A_{2}(r) \text { for all } r \in(R, \tilde{R}),
\end{aligned}
$$

then $\left(x_{2}, \Omega_{2}, M\right)$ is initially strictly hotter than $\left(x_{1}, \Omega_{1}, M\right)$.

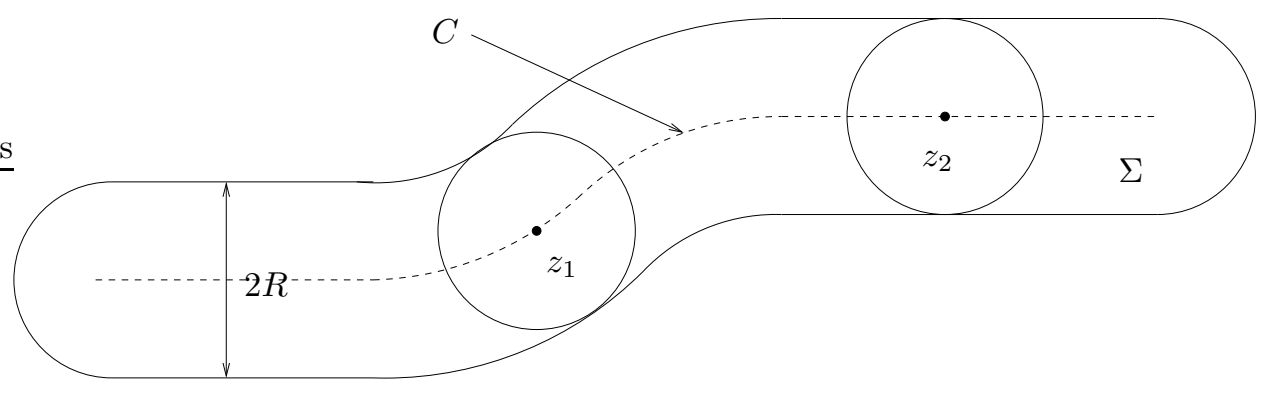

Figure 3. Example 6: Comparison of $\left(z_{1}, \Sigma, \mathbb{R}^{2}\right)$ and $\left(z_{2}, \Sigma, \mathbb{R}^{2}\right)$ 


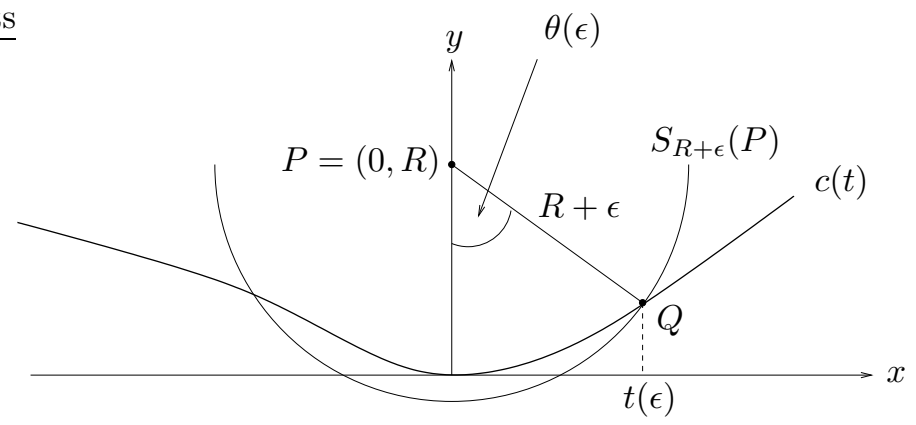

Figure 4. Asymptotics of the angle $\theta(\epsilon)$

Two points $x_{1} \in \Omega_{1}$ and $x_{2} \in \Omega_{2}$ with the same distance $R>0$ from the boundaries can thus be compared via the behavior of the corresponding spherical area functions on the interval $(R, \tilde{R})$.

Before we return to Examples 5 and 6 we first discuss the asymptotics of a particular angle (see Figure 4) in a useful model case.

Proposition 1.9. Let $\kappa \in \mathbb{R}$ be a constant and $c: \mathbb{R} \rightarrow \mathbb{R}^{2}$ be a curve passing horizontally through the origin and given by

$$
c(t)=t(1,0)+\frac{t^{2}}{2}(0, \kappa+\varphi(t))
$$

with $\lim _{t \rightarrow 0} \varphi(t)=0$. (Note that $\kappa$ is the curvature of $c$ at $t=0$.) Let $O$ denote the origin and $P$ denote the point $(0, R) \in \mathbb{R}^{2}$ for a fixed $R \in\left(0, \frac{1}{|k|}\right)$. Then the angle $\theta(\epsilon)=\angle O P Q$, given by the intersection point $Q$ of the circle $S_{R+\epsilon}(P)$ with

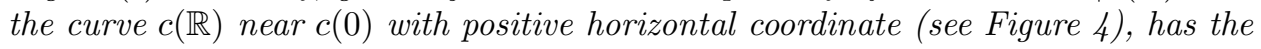
following asymptotics,

$$
\theta(\epsilon)=\frac{\sqrt{2 R}}{R+\epsilon}\left(\frac{1}{\sqrt{1-R \kappa}}+\psi(\epsilon)\right) \epsilon^{1 / 2}
$$

with $\lim _{\epsilon \rightarrow 0^{+}} \psi(\epsilon)=0$.

With Theorem 2 and Proposition 1.9 in hand, we can compare the initial heat of the points in Examples 5 and 6.

Examples 5 and 6 (continued): In Figure 2 the triple $\left(x_{1}, \Omega_{1}, \mathbb{R}^{2}\right)$ is initially strictly hotter than $\left(x_{2}, \Omega_{2}, \mathbb{R}^{2}\right)$, since $A_{1}(r)=A_{2}(r)$ for $0<r \leq R$, and $A_{1}(r)>$ $A_{2}(r)$ for $R<r<R+\delta$.

In Example 6, let $c:[a, b] \rightarrow \mathbb{R}^{2}$ be a parametrization of $C$. We choose a point $z=c(t) \in C$ with corresponding curvature $\kappa \in[0, k]$ (the arguments for the case $\kappa \in[-k, 0)$ go analogously). Then $S_{R}(z) \subset \Sigma$ touches $\partial \Sigma$ in two points where $\partial \Sigma$ has curvatures $0 \leq \frac{\kappa}{1+R \kappa} \leq \frac{\kappa}{1-R \kappa}$. Proposition 1.9 tells us that the corresponding spherical area function satisfies

$$
A(R+\epsilon)=2 \pi(R+\epsilon)-\sqrt{8 R}(\sqrt{1-R \kappa}+\sqrt{1+R \kappa}+o(1)) \epsilon^{1 / 2} .
$$

(Note that, seen from the point $z$ of the central curve $C$, the proposition has to be applied with the curvatures $\kappa_{-}=-\frac{\kappa}{1-R \kappa}$ and $\kappa_{+}=\frac{\kappa}{1+R \kappa}$.) 
For two points $z_{1}, z_{2} \in C$ with corresponding absolute curvatures $0 \leq \kappa_{1}<\kappa_{2} \leq$ $\kappa$ and corresponding spherical area functions $A_{i}(r)$ we conclude from the concavity of $x \mapsto \sqrt{x}$ that

$$
A_{1}(R+\epsilon)<A_{2}(R+\epsilon)
$$

for small enough $\epsilon>0$. Thus, $z_{2}$ is initially strictly hotter than $z_{1}$. The corresponding problem for a tube about a space curve $C \subset \mathbb{R}^{3}$ is discussed in detail in Appendix B

The following proposition treats the limiting behavior of the temperature at boundary points, as $t \rightarrow 0$.

Proposition 1.10. Let $\Omega \subset M$ be an admissible subset, $x \in \partial \Omega$ be a boundary point, $S_{r}(x)$ be the metric sphere of radius $r>0$ about $x$ and $A:[0, \infty) \rightarrow[0, \infty)$ be the associated spherical area function, i.e., $A(r)=\operatorname{vol}_{n-1}\left(S_{r}(x) \cap \Omega\right)$. Assume that the limit on the right hand side of (4) exists. Then the temperature limit at $x$ is given by

$$
\lim _{t \rightarrow 0^{+}} f_{\Omega, M}(t, x)=\lim _{r \rightarrow 0} \frac{A(r)}{\operatorname{vol}_{n-1}\left(S_{r}(x)\right)} .
$$

At smooth boundary points $x \in \partial \Omega$, we have, in particular,

$$
\lim _{t \rightarrow 0^{+}} f_{\Omega, M}(t, x)=\frac{1}{2} .
$$

Example 7: Let $\Pi$ be an arbitrary polygon in the Euclidean or hyperbolic plane $M$ with angles $\alpha_{1}, \alpha_{2}, \ldots, \alpha_{n}$ at the vertices $x_{1}, x_{2}, \ldots, x_{n}$, respectively. Then we have

$$
\lim _{t \rightarrow 0^{+}} f_{\Delta, M}(t, x)= \begin{cases}1 / 2 & \text { if } x \in \partial \Delta \backslash\left\{x_{1}, x_{2}, \ldots, x_{n}\right\}, \\ \alpha_{j} /(2 \pi) & \text { if } x=x_{j} .\end{cases}
$$

As a refinement of the boundary behavior at smooth points we have the following consequence of Theorem 2

Corollary 1.11. Let $\Omega \subset \mathbb{R}^{n}$ be an admissible subset and let $H_{\partial \Omega}(x)$ denote the mean curvature of $\partial \Omega$ at a smooth point $x \in \partial \Omega$, with respect to the outer unit normal vector. Then we have

$$
H_{\partial \Omega}\left(z_{1}\right)<H_{\partial \Omega}\left(z_{2}\right) \Longrightarrow\left(z_{1}, \Omega, \mathbb{R}^{n}\right) \succ\left(z_{2}, \Omega, \mathbb{R}^{n}\right) .
$$

Let us, finally, discuss a heat comparison result based on Steiner symmetrization in $M=\mathbb{R}^{n}$ or $M=\mathbb{H}^{n}$. Steiner symmetrization is a geometric procedure which associates, to every compact set $A \subset M$, a new set $\mathcal{S}(A) \subset M$ of the same volume which is symmetric with respect to a given hyperplane $E \subset M$. This geometric procedure has many useful applications in isoperimetric problems. In this article we use the notions introduced in Pey-02.

Definition 1.12. Let $M=\mathbb{R}^{n}$ or $M=\mathbb{H}^{n}, g \subset M$ be a geodesic and $E$ be a orthogonal hyperplane to $\mathrm{g} . h$ is called a $g$-line if there exists a 2-plane containing both $g$ and $h$ such that $h$ is a curve of fixed distance to $g$. Let $\pi: M \rightarrow E$ denote the projection whose preimages are the g-lines.

Let $A \subset M$ be a compact set. Steiner symmetrization $\mathcal{S}(A) \subset M$ with respect to the data $(g, E)$ is then uniquely determined by the following properties:

(a) For all g-lines $h$, the intersection $\mathcal{S}(A) \cap h$ is either empty or a bounded closed interval which is symmetric with respect to $E$. 
(b) If, for every g-line $h, \lambda_{h}$ denotes the Riemannian measure of the submanifold $h \subset M$, then we have

$$
\lambda_{h}(\mathcal{S}(A) \cap h)=\lambda_{h}(A \cap h) .
$$

Moreover, $\mathcal{S}(A) \cap h$ is empty if and only if $A \cap h$ is empty.

In the Euclidean case, $g$-lines are just straight Euclidean lines parallel to $g$. If $M=\mathbb{H}^{2}, g$-lines are hypercycles with the same end points as $g$.

Steiner symmetrization enjoys the following useful properties (see, e.g., Pey-02. Prop. 8]):

(S1) $A \subset B$ implies that $\mathcal{S}(A) \subset \mathcal{S}(B)$.

(S2) We have $\operatorname{vol}_{n}(\mathcal{S}(A))=\operatorname{vol}_{n}(A)$.

(S3) If $B \subset M$ is a closed metric ball of radius $R>0$ about $x \in M$, then $\mathcal{S}(B)$ is a closed metric ball of the same radius about $\pi(x)$.

Our last result reads as follows:

Proposition 1.13. Let $M=\mathbb{R}^{n}$ or $M=\mathbb{H}^{n}, \mathcal{S}$ denote Steiner symmetrization in $M$ with respect to the data $(g, E)$ and $\pi: M \rightarrow E$ denote orthogonal projection along g-lines. Then we have for every compact admissible set $\Omega \subset M$ and every point $x \in \Omega$ :

$$
f_{\Omega, M}(t, x) \leq f_{\mathcal{S}(\Omega), M}(t, \pi(x)) \quad \text { for all times } t>0 .
$$

Let $E_{x}$ be the orthogonal hyperplane to $g$ through $x$ and $s_{x}: M \rightarrow M$ denote the reflection in $E_{x}$. If $\Omega$ is not essentially symmetric with respect to $E_{x}$, i.e.,

$$
\operatorname{vol}_{n}\left(\Omega \Delta s_{x}(\Omega)\right)>0,
$$

then the above inequality (15) holds strictly.
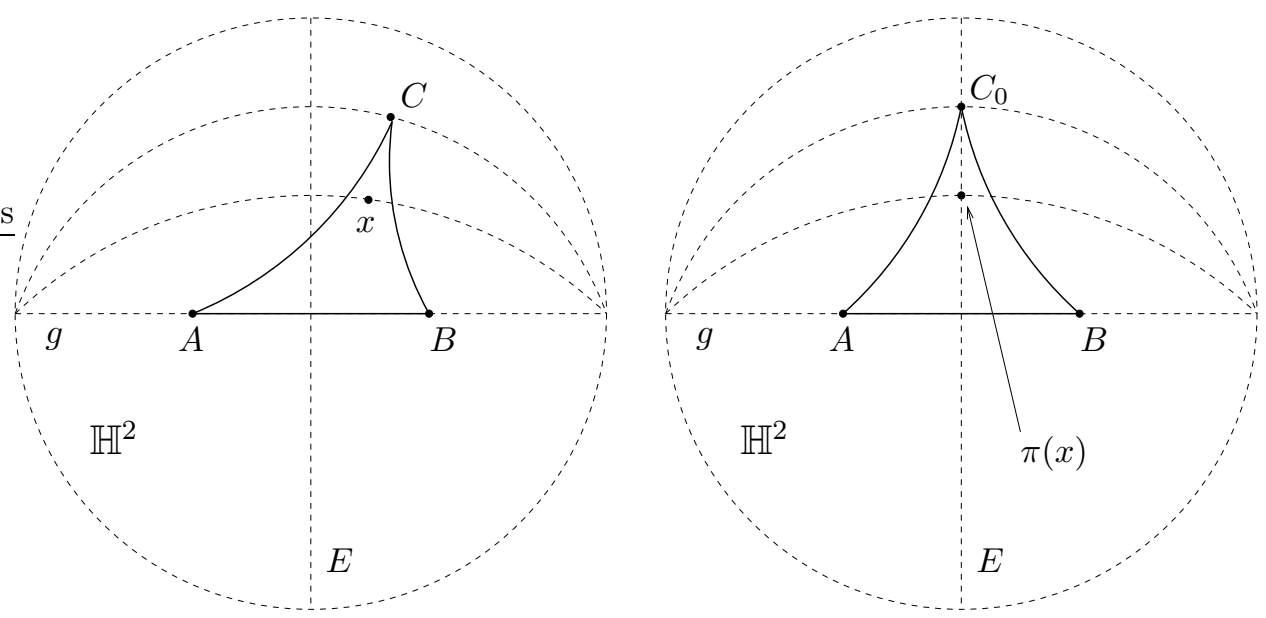

Figure 5. Example 8: Heat comparison of hyperbolic triangles with the same base and of the same height

Let us illustrate this result in an example.

Example 8: Let $M=\mathbb{R}^{2}$ or $M=\mathbb{H}^{2}$. Let $A B$ be a finite interval of a geodesic $g$ in $M, E \subset \mathbb{H}^{2}$ be the perpendicular bisector of $A B$ and $\pi: M \rightarrow E$ be the orthogonal 
projection along $g$-lines. Let $C$ be a point outside $E$ and $C_{0}=\pi(C) \in E$. The two triangles $\Delta_{0}=\triangle A B C_{0}$ and $\Delta=\triangle A B C$ have the same base and the same height and $\Delta_{0}$ is isosceles (see Figure 5 for the case $M=\mathbb{H}^{2}$ ). Then we have for any point $x \in \Delta$ :

$$
f_{\Delta, M}(t, x)<f_{\Delta_{0}, M}(t, \pi(x)) \text { for all times } t>0 .
$$

This can be seen as follows: Proposition 1.13 implies that

$$
f_{\Delta, M}(t, x)<f_{\mathcal{S}(\Delta), M}(t, \pi(x)) \text { for all times } t>0 .
$$

If $M=\mathbb{R}^{2}$, we have $\mathcal{S}(\Delta)=\Delta_{0}$ and we are done. It remains to consider the case $M=\mathbb{H}^{2}$ : In [KP-02, Thm. 4], we proved that $\mathcal{S}(\Delta)$ is strictly contained in $\Delta_{0}$ (see also Gue-03 for an easier proof of this fact). Positivity of the heat kernel implies strict domain monotonicity of the temperature, i.e., we have

$$
f_{\mathcal{S}(\Delta), \mathbb{H}^{2}}(t, y)<f_{\Delta_{0}, \mathbb{H}^{2}}(t, y) \quad \text { for all } y \in \mathbb{H}^{2} \text { and all times } t>0 \text {. }
$$

Choosing $y=\pi(x)$ finishes the proof of inequality (6) also in the hyperbolic case.

A comparison result, based on symmetrization, for solutions of more general parabolic equations is given, e.g., in ALT-91.

At the end of the Introduction we like to give a brief explanation of the structure of this article. In the next section we prove Theorems 1 and 2 Section 3 presents the proofs of all the other corollaries and propositions of this Introduction. The article ends with two appendices discussing heat properties in further examples and an appendix discussing an application of the Principle of not feeling the boundary.

Acknowledgements: The authors like to thank Oliver Stein and Djoko Wirosoetisno for helpful discussions.

\section{Proof of the Main Results}

In this section we present the proofs of the two theorems of the Introduction. In each lemma, proposition and corollary of this section the geometric requirements on the underlying manifolds are explicitely stated.

All our results are derived from particular properties of the heat kernel. Classical textbook accounts about heat kernels are, e.g., BGM-72, Cha-84, Gri-99, SchY-94. Some fundamental properties of heat kernels are listed in the following proposition.

Proposition 2.1. Let $M$ be a complete Riemannian manifold with lower Ricci curvature bound. Then there exists a unique smooth heat kernel

$$
k_{M}:(0, \infty) \times M \times M \rightarrow \mathbb{R}
$$

with the following properties:

(HK1) (positivity) We have $k_{M}>0$ on $(0, \infty) \times M \times M$.

(HK2) (heat conservation) We have, for all $(t, x) \in(0, \infty) \times M$ :

$$
\int_{M} k_{M}(t, x, y) d y=1
$$

(HK3) In the case $M=\mathbb{R}^{n}$ or $M=\mathbb{H}^{n}$ there is a strictly decreasing function $g_{M}:[0, \infty) \rightarrow \mathbb{R}$ such that $k_{M}(t, x, y)=g_{M}(d(x, y))$. 

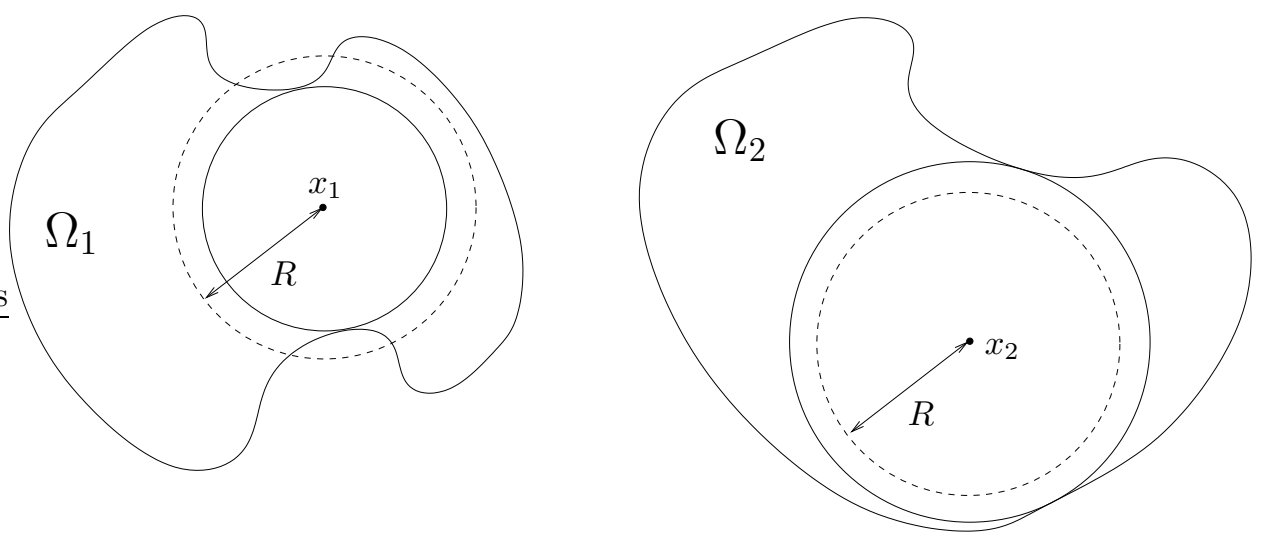

FIGURE 6. Introduction of the comparison triple $\left(x_{1}, B_{R}\left(x_{1}\right), M_{1}\right)$

(HK4) In the case $M=\mathbb{R}^{n}$ we have, for any choice $r_{1}<r_{2}<r_{3}$ of radii, a constant $\tau_{0}>0$, such that

$$
\int_{M \backslash B_{r_{3}}(x)} k_{M}(t, x, y) d y<\int_{B_{r_{2}}(x) \backslash B_{r_{1}}(x)} k_{M}(t, x, y) d y, \quad \text { for all } t \in\left(0, \tau_{0}\right) .
$$

Proof. (HK1), (HK2) and (HK3) are well known facts, see, e.g., Cha-84 pages 181,191,192]. Multiple applications of integration by parts yield the inequality

$$
\int_{M \backslash B_{r_{3}}(x)} k_{M}(t, x, y) d y \leq \frac{p(t)}{(4 \pi t)^{n / 2}} e^{-r_{3}^{2} /(4 t)},
$$

where $p$ is a polynomial with coefficients only depending on $n$ and $r_{3}$. (If $n$ is odd, (77) holds with equality; if $n$ is even, we first use the estimate

$$
\int_{r_{3}}^{\infty} r^{n} e^{-r^{2} /(4 t)} d r \leq \frac{1}{r_{3}} \int_{r_{3}}^{\infty} r^{n+1} e^{-r^{2} /(4 t)} \text {.) }
$$

On the other hand we have

$$
\int_{B_{r_{2}}(x) \backslash B_{r_{1}}(x)} k_{M}(t, x, y) d y \geq \frac{C}{(4 \pi t)^{n / 2}} e^{-r_{2}^{2} /(4 t)}
$$

with a fixed constant $C>0$ only depending on $n, r_{1}$ and $r_{2}$. Both estimates (7) and (8) immediately imply property (HK4).

Remark: We will show that (HK4) generalizes to arbitrary Riemannian manifolds, see Corollary 2.4 below. Property (HK4) is the key observation in this article.

For the proof of Theorem 1 we introduce a third comparison triple $\left(x_{1}, B_{R}\left(x_{1}\right), M_{1}\right)$ and thus break down the statement of the theorem into two smaller results which are presented in the Propositions A and B below (see Figure 6). We first state these propositions without proof:

Proposition A. Consider the situation in Theorem $\square$ and let $R \in\left(R_{1}, R_{2}\right)$. Then there exists a $\tau_{A}>0$ such that

$$
f_{B_{R}\left(x_{1}\right), M_{1}}\left(x_{1}, t\right)<f_{B_{R_{2}}\left(x_{2}\right), M_{2}}\left(x_{2}, t\right) \quad \text { for all } x_{1} \in I_{1}, x_{2} \in I_{2} \text { and } t \in\left(0, \tau_{A}\right) \text {. }
$$


Proposition B. Consider the situation in Theorem 1 and let $R \in\left(R_{1}, R_{2}\right)$. Then there exists a $\tau_{B}>0$ such that

$$
f_{\Omega_{1}, M_{1}}\left(x_{1}, t\right)<f_{B_{R}\left(x_{1}\right), M_{1}}\left(x_{1}, t\right) \quad \text { for all } x_{1} \in I_{1} \text { and } t \in\left(0, \tau_{B}\right) \text {. }
$$

Proof of Theorem 1. It is sufficient to prove the general statement of the theorem about the sets $I_{1}$ and $I_{2}$. We choose $R \in\left(R_{1}, R_{2}\right)$. Let $0<t<\min \left\{\tau_{A}, \tau_{B}\right\}$ and $x_{1} \in I_{1}$ and $x_{2} \in I_{2}$ be given. With the Propositions A and B we conclude that

$$
f_{\Omega_{1}, M_{1}}\left(x_{1}, t\right)<f_{B_{R}\left(x_{1}\right), M_{1}}\left(x_{1}, t\right)<f_{B_{R_{2}}\left(x_{2}\right), M_{2}}\left(x_{2}, t\right) \leq f_{\Omega_{2}, M_{2}}\left(x_{2}, t\right),
$$

where the last inequality follows from domain monotonicity $B_{R_{2}}\left(x_{2}\right) \subset \Omega_{2}$. This finishes the proof.

The proofs of Propositions A and B are a consequence of a sequence of lemmata, which we discuss next.

Lemma 2.2. Let $M_{2}$ be a complete Riemannian manifold with lower Ricci curvature bound $-\kappa<0$, upper sectional curvature bound $K>0$ and positive lower bound $i_{0}$ on the injectivity radius. Then there exists, for every small $\epsilon>0$, a constant $C_{u}>0$ and a time $\tau_{1}>0$, both only depending on $\operatorname{dim} M, \kappa, K, i_{0}, R$ and $\epsilon$ such that

$$
\int_{M_{2} \backslash B_{R}(x)} k_{M_{2}}(t, x, y) d y \leq C_{u} e^{-(R-\epsilon)^{2} /(4 t)},
$$

for all $t \in\left(0, \tau_{1}\right)$ and $x \in M_{2}$.

Proof. We assume that $\alpha<1 / 4$ is a constant close to $1 / 4$. We will see later how $\alpha$ has to be chosen. By the heat kernel estimate of Li and Yau (see [LY-86. Cor. 3.1]), we have

$$
\begin{aligned}
k_{M}(t, x, y) & \leq \frac{C_{1} e^{C_{2} t}}{\left(\operatorname{vol}\left(B_{\sqrt{t}}(x)\right) \operatorname{vol}\left(B_{\sqrt{t}}(y)\right)\right)^{1 / 2}} \exp \left(-\frac{\alpha d^{2}(x, y)}{t}\right) \\
& \leq \frac{C_{3}}{t^{n / 2}} \exp \left(-\frac{\alpha d^{2}(x, y)}{t}\right),
\end{aligned}
$$

for all $t \in\left(0, \sqrt{i_{0} / 2}\right]$ and $x, y \in M_{2}$. The constants $C_{1}, C_{2}>0$ depend only on $\alpha, \kappa$ and $n=\operatorname{dim} M$, whereas the existence of $C_{3}>0$ follows from Bishop/Günther and depends also on the upper sectional curvature bound $K>0$. Now, the volume form $d \mathrm{vol}=\rho(r, \theta) d \theta d r$ in geodesic polar coordinates about $x \in M_{2}$ is defined on a star-shaped subset of $T_{x} M_{2}$ and satisfies

$$
\rho(r, \theta) \leq C_{4} e^{C_{5} r}, \quad \text { with } C_{5}=(n-1) \sqrt{-\kappa} .
$$


Estimating the integral in (9) by an integration in $T_{x} M_{2}$, we obtain

$$
\begin{gathered}
\int_{M_{2} \backslash B_{R}(x)} k_{M_{2}}(t, x, y) d y \leq \frac{C_{3}}{t^{n / 2}} \int_{R}^{\infty} \int_{S^{n-1}} \exp \left(-\frac{\alpha r^{2}}{t}\right) \rho(r, \theta) d \theta d r \\
\leq \frac{C_{6}}{t^{n / 2}} \int_{R}^{\infty} \exp \left(-\frac{\alpha r^{2}}{t}+C_{5} r\right) d r \\
\leq \frac{C_{6}}{t^{n / 2}} \exp \left(\frac{C_{5}^{2} t}{4 \alpha}\right) \int_{R}^{\infty} \frac{r-\left(C_{5} t\right) /(2 \alpha)}{R-\left(C_{5} t\right) /(2 \alpha)} \exp \left(-\frac{\alpha}{t}\left(r-\frac{C_{5} t}{2 \alpha}\right)^{2}\right) d r \\
\leq \frac{C_{7}}{t^{n / 2}} \exp \left(-\frac{\alpha}{t}\left(R-\frac{C_{5} t}{2 \alpha}\right)^{2}\right) .
\end{gathered}
$$

For $\epsilon>0$ given, we can choose $0<\epsilon_{0}<\epsilon$ and $\alpha$ close enough to $1 / 4$, right at the beginning, such that there is a time $\tau_{1} \in\left(0, \sqrt{i_{0} / 2}\right)$ with

$$
\begin{aligned}
\frac{1}{t^{n / 2}} \exp \left(-\frac{\alpha}{t}\left(R-\frac{C_{5} t}{2 \alpha}\right)^{2}\right) \leq \frac{1}{t^{n / 2}} & \exp \left(-\frac{\left(R-\epsilon_{0}\right)^{2}}{4 t}\right) \\
\leq & \exp \left(-\frac{(R-\epsilon)^{2}}{4 t}\right), \text { for all } t \in\left(0, \tau_{1}\right) .
\end{aligned}
$$

Note that all constants $C_{j}>0$ in this proof are positive and depend only on the parameters mentioned in the lemma.

Lemma 2.3. Let $M$ be a complete Riemannian manifold with Ricci curvature bounded from below and $I \subset M$ be a compact subset. Let $R, \delta>0$ be given such that $R+\delta<\operatorname{inj}(x)$ for all $x \in I$. Then there exists a constant $C_{l}>0$ and a time $\tau_{2}>0$ such that

$$
\int_{B_{R+\delta}(x) \backslash B_{R}(x)} k_{M}(t, x, y) d y \geq C_{l} e^{-(R+\delta)^{2} /(4 t)},
$$

for all $t \in\left(0, \tau_{2}\right)$ and $x \in I$.

Proof. Let $U \subset M$ denote the open $(R+2 \delta)$-tube about $I$ and $k_{U}^{D}$ denote the corresponding Dirichlet heat kernel. By the Minakshisundaram-Pleijel expansion there is a smooth function $u_{0}$ such that we have for all $x \in I$ and $y \in B_{R+\delta}(x)$,

$$
k_{U}^{D}(t, x, y)=\left(u_{0}(x, y)+O(t)\right) \frac{1}{(4 \pi t)^{n / 2}} \exp \left(-\frac{d^{2}(x, y)}{4 t}\right), \quad \text { as } t \rightarrow 0
$$

with a uniform $O(t)$. Here, $u_{0}(x, y)=\varphi^{-1 / 2}(x, y)$ (see Cha-84]), where $\varphi$ is a density function satisfying

$$
\int_{B_{R}(y)} f(x) d x=\int_{0}^{R} \int_{S_{y} M} f\left(\exp _{y}(t v)\right) \varphi\left(\exp _{y}(t v), y\right) d \operatorname{vol}_{S_{y} M}(v) d t,
$$

for all $f \in L^{1}\left(B_{R}(y)\right)$. Note that, by construction, $k_{U}^{D} \leq k_{M}$. Therefore, for all $x, y$ as above there exists a $\hat{\tau}>0$ such that

$$
k_{M}(t, x, y) \geq \frac{1}{2} u_{0}(x, y) \frac{1}{(4 \pi t)^{n / 2}} \exp \left(-\frac{d^{2}(x, y)}{4 t}\right),
$$


for all $t \in(0, \hat{\tau})$. It follows that

$$
\begin{aligned}
\int_{B_{R+\delta}(x) \backslash B_{R}(x)} k_{M}(t, x, y) d y & =\int_{R}^{R+\delta} \int_{S_{r}(x)} k_{M}(x, y, t) d \operatorname{vol}_{S_{r}(x)}(y) d r \\
\geq & \frac{1}{(4 \pi t)^{n / 2}} \int_{R}^{R+\delta} e^{-r^{2} /(4 t)} \int_{S_{r}(x)} \frac{u_{0}(x, y)}{2} d \operatorname{vol}_{S_{r}(x)}(y) d r .
\end{aligned}
$$

Since there exists a $C_{0}>0$ such that

$$
\int_{S_{r}(x)} u_{0}(x, y) d \operatorname{vol}_{S_{r}(x)}(y) d r \geq C_{0},
$$

for all $x \in I$ and $r \in[R, R+\delta]$, we can find a $\tau_{2} \in(0, \hat{\tau})$ such that

$$
\int_{B_{R+\delta}(x) \backslash B_{R}(x)} k_{M}(t, x, y) d y \geq \frac{\delta C_{0}}{2} \frac{e^{-(R+\delta)^{2} /(4 t)}}{(4 \pi t)^{n / 2}} \geq C_{l} e^{-(R+\delta)^{2} / 4 t},
$$

for all $t \in\left(0, \tau_{2}\right)$.

A consequence of the previous lemmata is the following result, generalizing property (HK4) to arbitrary Riemannian manifolds.

Corollary 2.4. Let $M$ be a complete Riemannian manifold with lower Ricci curvature bound, upper sectional curvature bound and lower positive bound on the injectivity radius. Let $I \subset M$ be a compact subset and $0 \leq R_{0}<\tilde{R}$ with $\operatorname{inj}(x)>R_{0}$ for all $x \in I$. Then there exists, for any $0<\delta<\tilde{R}-R_{0}$ and every $\eta>0, a \tau_{0}>0$ such that we have

$$
\int_{M \backslash B_{\tilde{R}}(x)} k_{M}(t, x, y) d y<\eta \int_{B_{R_{0}+\delta}(x) \backslash B_{R_{0}}(x)} k_{M}(t, x, y) d y,
$$

for all $t \in\left(0, \tau_{0}\right)$ and all $x \in I$.

Proof. Without loss of generality, we may assume that $R_{0}+\delta<\operatorname{inj}(x)$ for all $x \in I$. Now, choose $\epsilon>0$ such that $R_{0}+\delta<\tilde{R}-\epsilon$. Then we can conclude with the help of Lemmata 2.2 and 2.3 that there is a $\tau_{0} \in\left(0, \min \left\{\tau_{1}, \tau_{2}\right\}\right)$ such that

$$
\begin{aligned}
\int_{M \backslash B_{\tilde{R}}(x)} k_{M}(t, x, y) d y & \leq C_{u} e^{-(\tilde{R}-\epsilon)^{2} /(4 t)} \\
& <\eta C_{l} e^{-\left(R_{0}+\delta\right)^{2} /(4 t)} \leq \eta \int_{B_{R_{0}+\delta}(x) \backslash B_{R_{0}}(x)} k_{M}(t, x, y) d y,
\end{aligned}
$$

for all $t \in\left(0, \tau_{0}\right)$ and all $x \in I$.

Next, we prove Proposition A:

Proof of Proposition A. Choose $\delta, \epsilon>0$ such that $R_{1}+\delta<\min \left\{\operatorname{inj}\left(x_{1}\right), R_{2}-\epsilon\right\}$ for all $x_{1} \in I_{1}$. We conclude from (HK2) and Lemmata 2.2 and 2.3 that there is a $\tau_{A} \in\left(0, \min \left\{\tau_{1}, \tau_{2}\right\}\right)$ such that we have, for all $x_{1} \in I_{1}, x_{2} \in I_{2}$ and $t \in\left(0, \tau_{A}\right)$,

$$
\begin{aligned}
\int_{B_{R_{1}}\left(x_{1}\right)} k_{M_{1}}\left(t, x_{1}, y\right) d y & =1-\int_{M_{1} \backslash B_{R_{1}}\left(x_{1}\right)} k_{M_{1}}\left(t, x_{1}, y\right) d y \\
\leq & 1-C_{l} e^{-\left(R_{1}+\delta\right)^{2} /(4 t)}<1-C_{u} e^{-\left(R_{2}-\epsilon\right)^{2} /(4 t)} \\
& \leq 1-\int_{M_{2} \backslash B_{R_{2}}\left(x_{2}\right)} k_{M_{2}}\left(t, x_{2}, y\right) d y=\int_{B_{R_{2}}\left(x_{2}\right)} k_{M_{2}}\left(t, x_{2}, y\right) d y
\end{aligned}
$$


This finishes the proof of Proposition A.

The proof of Proposition B is based on the following fact:

Lemma 2.5 (Rearrangement-Lemma). Assume that there are two non-negative functions $A_{1}, A_{2}:[0, \rho] \rightarrow \mathbb{R}$ satisfying the following properties:

i) $A_{1}(r) \leq A_{2}(r)$, for all $r \in[0, \rho]$,

i)) $\exists \rho_{0} \in[0, \rho)$ with $\int_{0}^{\rho} A_{1}(r) d r<\int_{0}^{\rho_{0}} A_{2}(r) d r$.

Then we have, for every non-increasing function $f:[0, \rho] \rightarrow(0, \infty)$ :

$$
\int_{0}^{\rho} A_{1}(r) f(r) d r<\int_{0}^{\rho_{0}} A_{2}(r) f(r) d r
$$

Proof. We have

$$
\begin{gathered}
\int_{0}^{\rho} A_{1}(r) f(r) d r=\int_{0}^{\rho_{0}} A_{1}(r) f(r) d r+\int_{\rho_{0}}^{\rho} A_{1}(r) f(r) d r \\
\quad=\int_{0}^{\rho_{0}} A_{2}(r) f(r) d r+\int_{0}^{\rho_{0}} \underbrace{\left(A_{1}(r)-A_{2}(r)\right.}_{\leq 0} f(r) d r+\int_{\rho_{0}}^{\rho} A_{1}(r) f(r) d r \\
\quad \leq \int_{0}^{\rho_{0}} A_{2}(r) f(r) d r+f\left(\rho_{0}\right) \int_{0}^{\rho_{0}} A_{1}(r)-A_{2}(r) d r+f\left(\rho_{0}\right) \int_{\rho_{0}}^{\rho} A_{1}(r) d r \\
=\int_{0}^{\rho_{0}} A_{2}(r) f(r) d r+f\left(\rho_{0}\right)\left(\int_{0}^{\rho} A_{1}(r) d r-\int_{0}^{\rho_{0}} A_{2}(r) d r\right)<\int_{0}^{\rho_{0}} A_{2}(r) f(r) d r .
\end{gathered}
$$

As a consequence of the Rearrangement-Lemma we have the following property of the heat kernel:

Lemma 2.6. Let $M$ be a complete Riemannian manifold with lower bound on the Ricci curvature, upper bound on the sectional curvature and lower positive bound on the injectivity radius. Let $\Omega \subset M$ be an admissible set and $R: M \rightarrow[0, \infty]$ be the corresponding distance from the complement. Let $I \subset M$ be compact and $R_{1}, \tilde{R}>0$ satisfy

$$
R(x) \leq R_{1}<\tilde{R}<\operatorname{inj}(x) \quad \text { for all } x \in I .
$$

Then there exists an $R_{0} \in\left(R_{1}, \tilde{R}\right)$ and a time $\tau_{3}>0$ such that

$$
\int_{\Omega \cap B_{\tilde{R}}(x)} k_{M}(t, x, y) d y<\int_{B_{R_{0}}(x)} k_{M}(t, x, y) d y,
$$

for all $t \in\left(0, \tau_{3}\right)$ and all $x \in I$.

Proof. Let

$$
u_{t}(x, y):=(4 \pi t)^{n / 2} e^{d^{2}(x, y) /(4 t)} k_{M}(t, x, y) .
$$

Applying Corollary [C.1] of Appendix [C] (with $\delta=\tilde{R}$ ) we have $u_{t}(x, z) \rightarrow u_{0}(x, z)$, uniformly on $B_{\tilde{R}}(x)$, as $t \rightarrow 0$. Here, $u_{0}$ is given by the Minakshisundaram-Pleijel expansion. Now we introduce the functions

$$
\begin{aligned}
A_{1}(t, r) & :=\int_{S_{r}(x) \cap \Omega} u_{t}(x, y) d \operatorname{vol}_{S_{r}(x)}(y), \\
A_{2}(t, r) & :=\int_{S_{r}(x)} u_{t}(x, y) d \operatorname{vol}_{S_{r}(x)}(y),
\end{aligned}
$$


for all small $t \geq 0$. Since $\tilde{R}>R(x)$, we have

$$
\int_{0}^{\tilde{R}} A_{1}(0, r) d r<\int_{0}^{\tilde{R}} A_{2}(0, r)
$$

Choosing an $R_{0} \in[R(x), \tilde{R})$ with

$$
\int_{0}^{\tilde{R}} A_{1}(0, r) d r<\int_{0}^{R_{0}} A_{2}(0, r) d r
$$

there is also a $\tau_{3}>0$ such that

$$
\int_{0}^{\tilde{R}} A_{1}(t, r) d r<\int_{0}^{R_{0}} A_{2}(t, r) d r
$$

for all $t \in\left[0, \tau_{3}\right)$. Now we apply the Rearrangement Lemma with the function $f_{t}(r)=\frac{1}{(4 \pi t)^{n / 2}} e^{-r^{2} /(4 t)}$ and obtain

$$
\begin{aligned}
\int_{\Omega \cap B_{\tilde{R}}(x)} k_{M}(t, x, y) d y & =\int_{0}^{\tilde{R}} \int_{S_{r}(x) \cap \Omega} k_{M}(t, x, y) d \operatorname{vol}_{S_{r}(x)}(y) d r \\
& <\int_{0}^{R_{0}} \int_{S_{r}(x)} k_{M}(t, x, y) d \operatorname{vol}_{S_{r}(x)}(y) d r=\int_{B_{R_{0}}(x)} k_{M}(t, x, y) d y
\end{aligned}
$$

for all $t \in\left(0, \tau_{3}\right)$.

Now we prove Proposition B:

Proof of Proposition B. We choose $\tilde{R} \in\left(R_{1}, R\right)$. We obviously have for all $x_{1} \in I_{1}$ :

$$
\int_{\Omega_{1}} k_{M_{1}}\left(t, x_{1}, y\right) d y \leq \int_{\Omega_{1} \cap B_{\tilde{R}}\left(x_{1}\right)} k_{M_{1}}\left(t, x_{1}, y\right) d y+\int_{M_{1} \backslash B_{\tilde{R}}\left(x_{1}\right)} k_{M_{1}}\left(t, x_{1}, y\right) d y .
$$

We conclude from Lemma 2.6 that there is an $R_{0} \in\left(R_{1}, \tilde{R}\right)$ and a time $\tau_{3}>0$ such that we have

$$
\int_{\Omega_{1} \cap B_{\tilde{R}}\left(x_{1}\right)} k_{M_{1}}\left(t, x_{1}, y\right) d y<\int_{B_{R_{0}}\left(x_{1}\right)} k_{M_{1}}\left(t, x_{1}, y\right) d y
$$

for all $x_{1} \in I_{1}$ and $t \in\left(0, \tau_{3}\right)$. Choosing $0<\delta<\tilde{R}-R_{0}$ we find, with the help of Corollary 2.4 a time $\tau_{0}>0$ such that

$$
\int_{M_{1} \backslash B_{\tilde{R}}\left(x_{1}\right)} k_{M_{1}}\left(t, x_{1}, y\right) d y<\int_{B_{R_{0}+\delta}\left(x_{1}\right) \backslash B_{R_{0}}\left(x_{1}\right)} k_{M_{1}}\left(t, x_{1}, y\right) d y,
$$

for all $x_{1} \in I_{1}$ and $t \in\left(0, \tau_{0}\right)$. Combining these facts we end up with

$$
\int_{\Omega_{1}} k_{M_{1}}\left(t, x_{1}, y\right) d y<\int_{B_{R_{0}+\delta}\left(x_{1}\right)} k_{M_{1}}\left(t, x_{1}, y\right) d y
$$

for all $x_{1} \in I_{1}$ and $t \in\left(0, \min \left\{\tau_{0}, \tau_{3}\right\}\right)$. Since $R_{0}+\delta<\tilde{R}<R$, this proves Proposition B.

The above lemmata enable us, finally, to present a relatively short proof of Theorem 2. 
Proof of Theorem [ We start with an obvious inequality and use (HK3) to obtain

$$
\begin{array}{r}
\int_{\Omega_{1}} k_{M}\left(t, x_{1}, y\right) d y \leq \int_{\Omega_{1} \cap B_{\tilde{R}}\left(x_{1}\right)} k_{M}\left(t, x_{1}, y\right) d y+\int_{M \backslash B_{\tilde{R}}\left(x_{1}\right)} k_{M}\left(t, x_{1}, y\right) d y \\
=\int_{0}^{\tilde{R}} g_{M}(r) A_{1}(r) d r+\int_{M \backslash B_{\tilde{R}}\left(x_{1}\right)} k_{M}\left(t, x_{1}, y\right) d y .
\end{array}
$$

The assumptions on $A_{1}, A_{2}$ and positivity of the heat kernel imply that there is an $R_{0} \in(R, \tilde{R})$ such that

$$
\int_{0}^{\tilde{R}} g_{M}(r) A_{1}(r) d r<\int_{0}^{R_{0}} g_{M}(r) A_{2}(r) d r
$$

Choose $\delta>0$ such that $R_{0}+\delta<\tilde{R}$. Since $A_{2}(r)>0$ for all $r \in\left[R_{0}, R_{0}+\delta\right]$ we can find an $\eta>0$ such that

$$
A_{2}(r)=\operatorname{vol}_{n-1}\left(S_{r}\left(x_{2}\right) \cap \Omega_{2}\right) \geq \eta \operatorname{vol}_{n-1}\left(S_{r}\left(x_{2}\right)\right), \quad \text { for all } r \in\left[R_{0}, R_{0}+\delta\right] .
$$

Corollary 2.4 implies that there exists a $\tau_{0}>0$ such that

$$
\begin{aligned}
\int_{M \backslash B_{\tilde{R}}\left(x_{1}\right)} k_{M}\left(t, x_{1}, y\right) d y<\eta \int_{R_{0}}^{R_{0}+\delta} g_{M}(r) \operatorname{vol}_{n-1}\left(S_{r}\left(x_{2}\right)\right) d r & \\
& \leq \int_{R_{0}}^{R_{0}+\delta} g_{M}(r) A_{2}(r) d r,
\end{aligned}
$$

for all $t \in\left(0, \tau_{0}\right)$. Putting these inequalities together we conclude that

$$
\int_{\Omega_{1}} k_{M}\left(t, x_{1}, y\right) d y<\int_{0}^{R_{0}+\delta} g_{M}(r) A_{2}(r) d r \leq \int_{\Omega_{2}} k_{M}\left(t, x_{2}, y\right) d y
$$

for all $t \in\left(0, \tau_{0}\right)$.

\section{Proof of the other Results of the Introduction}

Proof of Corollary 1.3. Let $\Gamma_{j}=\overline{\Omega_{j}^{c}}$ for $j=1,2$. Then $R_{j}^{-}$agree with the distances from the complements $\Gamma_{j}^{c}$. Applying Theorem 1 we conclude that

$$
\left(x_{1}, \Gamma_{1}, M_{1}\right) \succ\left(x_{2}, \Gamma_{2}, M_{2}\right) .
$$

Property $(\mathrm{HK} 2)$ and $\operatorname{vol}_{n}\left(\partial \Omega_{j}\right)=0$ imply

$$
\left(x_{2}, \Omega_{2}, M_{2}\right) \succ\left(x_{1}, \Omega_{1}, M_{1}\right),
$$

finishing the proof.

Corollary 1.5 is a trivial consequence of Theorem 1

Proof of Corollary [1.6. Let $f:(0, \infty) \times M \rightarrow \mathbb{R}$ denote the unique solution of (1).

It is sufficient to prove that the limit of every convergent subsequence of $x_{j}$ lies in $I_{\infty}(\Omega)$. So let us choose a convergent subsequence which we denote for simplicity, again, by $x_{j}$. By continuity of $R: \Omega \rightarrow[0, \infty)$ it suffices to prove that $R\left(x_{j}\right) \rightarrow R_{\infty}(\Omega)$. Let $\epsilon>0$ be an arbitrary small number. Choosing $I_{1}:=\{x \in$ $\left.\Omega \mid R(x) \leq R_{\infty}(\Omega)-\epsilon\right\}$ and $I_{2}:=I_{\infty}(\Omega)$, we conclude from Theorem 1 that there is a $\tau>0$ such that we have for all $0<t<\tau$,

$$
f\left(t, z_{1}\right)<f\left(t, z_{2}\right) \quad \text { for all } z_{1} \in I_{1}, z_{2} \in I_{2} \text {. }
$$


Consequently, we have $x_{j} \notin I_{1}$ for all $j$ with $t_{j}<\tau$. This finishes the proof of the corollary.

Proof of Proposition 1.7. The Euclidean heat kernel on $\mathbb{R}^{n}$ is given by

$$
k(t, x, y)=\frac{1}{(4 \pi t)^{n / 2}} e^{-(x-y)^{2} /(4 t)} .
$$

Let $\Omega \subset \mathbb{R}^{n}$ be compact with $\operatorname{vol}_{n}(\Omega)>0$ and $\tilde{\Omega}$ be its convex hull. By ChK-90. the maxima of $f:=f_{\Omega, \mathbb{R}^{n}}$ are located in $\tilde{\Omega}$.

We first prove that there is a $T>0$ such that

$$
f(t, x)=\int_{\Omega} k(t, x, y) d y
$$

is a concave function on $\tilde{\Omega}$ for all $t \geq T$. This immediately implies that $H(t) \subset \tilde{\Omega}$ consists of a single point for all $t \geq T$. An easy calculation shows that

$$
D_{x}^{2} k(t, x, y)=\frac{k(t, x, y)}{2 t} Q(t, x-y) \quad \text { with } Q(t, z)=\left(\frac{z_{i} z_{j}}{2 t}-\delta_{i j}\right)_{i j} .
$$

Note that $Q(t, z)$ can be considered as a perturbation of the negative definite matrix -Id. Since $\tilde{\Omega}$ is bounded, there is a $T>0$ such that $Q(t, x-y)$ is negative definite for all $x, y \in \tilde{\Omega}$ and all $t \geq T$. This implies for $t \geq T$ and $x \in \tilde{\Omega}$ that

$$
\left\langle D_{x}^{2} f(t, x) v, v\right\rangle=\int_{\Omega} \frac{k(t, x, y)}{2 t} \underbrace{\langle Q(t, x-y) v, v\rangle}_{<0} d y<0,
$$

i.e., $D_{x}^{2} f(t, x)$ is negative definite.

Since $D_{x} f: \mathbb{R} \times \mathbb{R}^{n} \rightarrow \mathbb{R}^{n}$ is smooth, $D_{x} f(t, H(t))=0$ and $\operatorname{det} D_{x}^{2} f(t, H(t)) \neq 0$ for $t \geq T$, the implicit function theorem tells us that the map $t \mapsto H(t)$ is smooth on $(T, \infty)$. Finally, we have

$$
\begin{aligned}
& \frac{\partial}{\partial t} f(t, H(t))=\frac{\partial f}{\partial t}(t, H(t))+\underbrace{\left\langle D_{x} f(t, H(t)), \dot{H}(t)\right\rangle}_{=0} \\
&=\Delta f(t, H(t))=\operatorname{tr} D_{x}^{2} f(t, H(t))<0 .
\end{aligned}
$$

Let $M$ be the Euclidean or the hyperbolic plane. Note that for every $t>0$ we can express the heat kernel $k_{M}$ by

$$
k_{M}(t, x, y)=g_{M}(d(x, y))
$$

with a strictly decreasing function $g_{M}:[0, \infty) \rightarrow \mathbb{R}$ (see property (HK3)). Proposition 1.8 follows now immediately from the following more general result (for a proof see, e.g., [FeT-73, or [Flo-93]):

Theorem 3 (Momentum lemma). Let $M$ be the Euclidean or the hyperbolic plane and $g:[0, \infty) \rightarrow \mathbb{R}$ be a strictly descreasing function. Let $\Sigma_{1}, \Sigma_{2} \subset M$ be two polygons of the same area and the same number of sides. Assume that $\Sigma_{1}$ is regular and that $x_{1}$ is its center. Then we have for all $x_{2} \in M$ :

$$
\int_{\Sigma_{1}} g\left(d\left(x_{1}, x\right)\right) d x \geq \int_{\Sigma_{2}} g\left(d\left(x_{2}, x\right)\right) d x
$$

and equality only holds if $\Sigma_{2}$ is also regular and if $x_{2}$ is the center of $\Sigma_{2}$. 
Proof of Proposition [1.9. The situation of the proposition is illustrated in Figure 4. Note that we have $|P-c(t)|>R$ for $t$ close enough to the origin 0 . Thus the curve $c(\mathbb{R})$ and the circle $S_{R+\epsilon}(P)$ intersect in two points, for small enough $\epsilon>0$, one of which is denoted by $Q$.

Pythagoras and the asymptotics of $x \mapsto \sqrt{1+x}$ imply that

$$
\epsilon(t)=|P-c(t)|-R=\frac{1-R \kappa}{2 R} t^{2}+o\left(t^{2}\right) .
$$

Thus we can also express $t$ as function of $\epsilon$ near 0 and obtain

$$
t(\epsilon)=\frac{\sqrt{2 R}}{\sqrt{1-R \kappa}} \epsilon^{1 / 2}+o\left(\epsilon^{1 / 2}\right)
$$

This implies that

$$
\theta(\epsilon) \sim \sin \theta(\epsilon)=\frac{t(\epsilon)}{R+\epsilon}=\frac{1}{R+\epsilon}\left(\frac{\sqrt{2 R}}{\sqrt{1-R \kappa}} \epsilon^{1 / 2}+o\left(\epsilon^{1 / 2}\right)\right),
$$

which proves the proposition.

Proof of Proposition 1.10 Let $n=\operatorname{dim} M$ and $r_{0}:=\operatorname{inj}(x)>0$. We conclude from Lemma 2.2 that

$$
\lim _{t \rightarrow 0^{+}} \int_{\Omega} k_{M}(t, x, y) d y=\lim _{t \rightarrow 0^{+}} \int_{\Omega \cap B_{r_{0} / 2}(x)} k_{M}(t, x, y) d y .
$$

Let $\omega_{n-1}$ denote the volume of the unit sphere in $\mathbb{R}^{n}$. Applying Corollary C.1] in Appendix $\mathbb{C}$ (with $I=\{x\}$ and $\delta=r_{0} / 2$ ) we obtain

$$
\begin{aligned}
& \lim _{t \rightarrow 0^{+}} \int_{\Omega \cap B_{r_{0} / 2}(x)} k_{M}(t, x, y) d y \\
& \quad=\lim _{t \rightarrow 0^{+}} \frac{1}{(4 \pi t)^{n / 2}} \int_{0}^{r_{0} / 2} e^{-\frac{r^{2}}{4 t}} \int_{S_{r}(x) \cap \Omega}\left(u_{0}(x, y)+O(t)\right) d \operatorname{vol}_{S_{r}(x)}(y) d r
\end{aligned}
$$

with a uniform $O(t)$ on the compact set $B_{r_{0} / 2}(x) \subset M$. Introducing

$$
g(r):=\frac{\int_{S_{r}(x) \cap \Omega} u_{0}(x, y) d \operatorname{vol}_{S_{r}(x)}(y)}{\omega_{n-1} r^{n-1}},
$$

we conclude that

$$
\begin{aligned}
\lim _{t \rightarrow 0^{+}} \frac{1}{(4 \pi t)^{n / 2}} \int_{0}^{r_{0} / 2} e^{-\frac{r^{2}}{4 t}} \int_{S_{r}(x) \cap \Omega}\left(u_{0}(x, y)+O(t)\right) d \operatorname{vol}_{S_{r}(x)}(y) d r \\
=\lim _{t \rightarrow 0^{+}} \frac{1}{(4 \pi t)^{n / 2}} \int_{0}^{\infty} e^{-\frac{r^{2}}{4 t}} \omega_{n-1} r^{n-1} g(r) d r \\
=\lim _{t \rightarrow 0^{+}} \int_{\mathbb{R}^{n}} k_{\mathbb{R}^{n}}(t, x, y) g(|x-y|) d y=\lim _{r \rightarrow 0} g(r) .
\end{aligned}
$$

The proposition follows now from

$$
\lim _{r \rightarrow 0} \frac{\operatorname{vol}_{n-1}\left(S_{r}(x)\right)}{\omega_{n-1} r^{n-1}}=1
$$

and the fact that $\lim _{r \rightarrow 0} u_{0}\left(x, \exp _{x}(r \xi)\right)=1$, uniformly for all $\xi$ in the tangent space $S_{x} M$. 


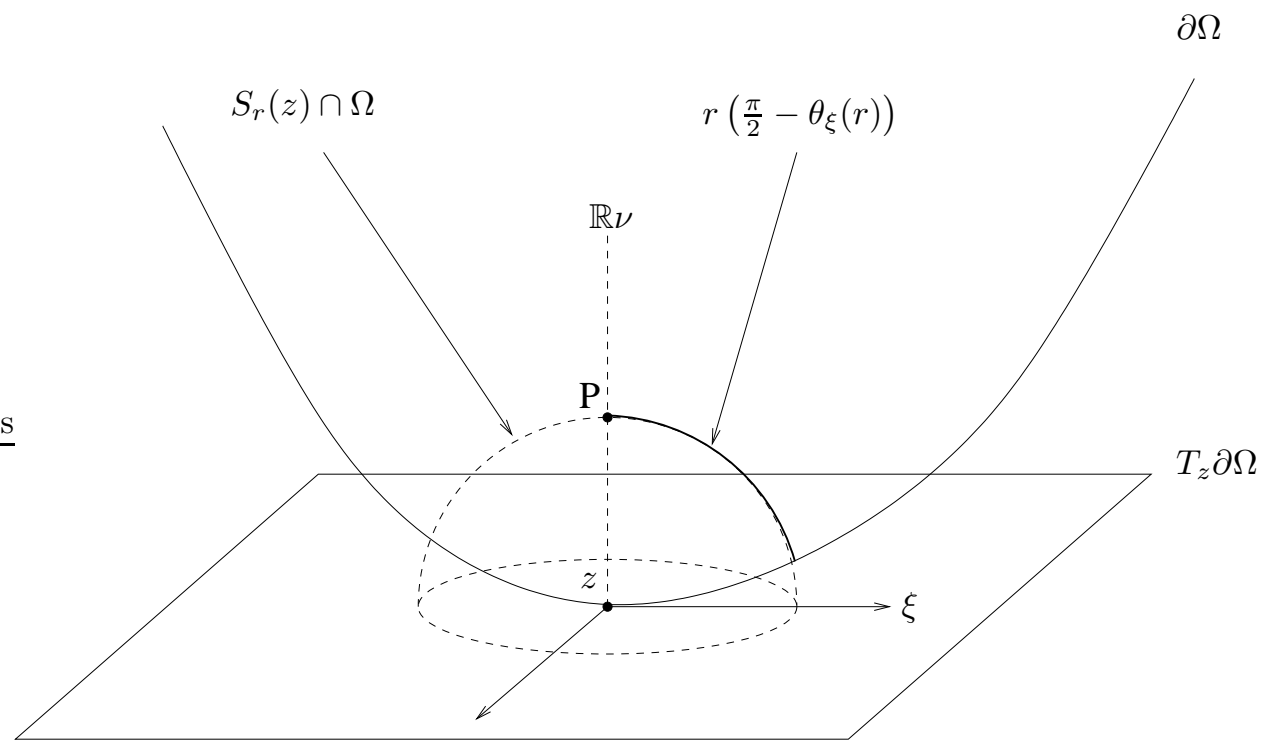

FiguRE 7. Calculation of $\operatorname{vol}_{n-1}\left(S_{r}(z) \cap \Omega\right)$

Proof of Corollary 1.11. The proof proceeds in two steps.

Step 1. We first consider the following model situation: Let $C \subset \mathbb{R}^{2}$ be a smooth planar curve through the origin $O$ with horizontal tangent and curvature $k$ at $O$. Then there exists, locally near $O$, a parametrization $c(r)=(x(r), y(r))$ of $C$ with $\dot{x}(r)>0$ and satisfying

$$
|c(r)|=r .
$$

This forces the Taylor expansions of the components $x(r), y(r)$ to be of the form

$$
\begin{aligned}
& x(r)=r(1+O(r)), \\
& y(r)=r^{2}\left(\frac{k}{2}+O(r)\right) .
\end{aligned}
$$

Let $\theta(r) \in[0, \pi / 2)$ denote the angle between the line through $O$ and $c(t)$ and the horizontal $x$-axis. The above expansions imply for the asymptotics of the angle $\theta(r)$ that

$$
\theta(r) \sim \tan \theta(r)=\frac{y(r)}{x(r)}=r\left(\frac{k}{2}+O(r)\right) .
$$

Step 2. Let $z \in \partial \Omega$ be a smooth boundary point and $L_{z}: T_{z} \partial \Omega \rightarrow T_{z} \partial \Omega$ denote the Weingarten map of $\partial \Omega$ at $z$ with respect to the outward unit normal vector $\nu$ of $\Omega$ in $z$. Using polar coordinates in $S_{r}(z)$ about the center $P=z-r \nu \in S_{r}(z)$ (see Figure 17) we conclude that

$$
\operatorname{vol}_{n-1}\left(S_{r}(z) \cap \Omega\right)=r^{n-1} \int_{S_{z} \partial \Omega} d \xi \int_{0}^{\frac{\pi}{2}-\theta_{\xi}(r)} \sin ^{n-2}(t) d t
$$


where $S_{z} \partial \Omega$ is the unit tangent space of $\partial \Omega$ in $z$ and canonically isometric to the standard unit sphere $S^{n-2}$ and $\theta_{\xi}$ is asymptotically given by

$$
\theta_{\xi}(r)=r\left(\frac{k(\xi)}{2}+O(r)\right)
$$

where $k(\xi)=\left\langle L_{z} \xi, \xi\right\rangle$ is the normal curvature of $\partial \Omega$ at $z$ in direction $\xi$, by Step 1 . Since

$$
\int_{0}^{\frac{\pi}{2}-\theta} \sin ^{n-2}(t) d t= \begin{cases}1-\sin (\theta)+\sum_{j=1}^{k} a_{j}\left(1-\sin ^{2 j+1}(\theta)\right) & \text { if } n-2=2 k+1, \\ b\left(\frac{\pi}{2}-\theta\right)-\sum_{j=1}^{k} b_{j} \sin (2 j \theta) & \text { if } n-2=2 k\end{cases}
$$

with suitable constants $a_{j}, b, b_{j}$ and $b, b_{j}>0$, we conclude that

$$
\int_{0}^{\frac{\pi}{2}-\theta_{\xi}(r)} \sin ^{n-2}(t) d t=C_{1}-C_{2} r \frac{k(\xi)}{2}+O\left(r^{2}\right)
$$

with suitable constants $C_{1}, C_{2}>0$. This implies that

$$
\begin{aligned}
A(r) & =\operatorname{vol}_{n-1}\left(S_{r}(z) \cap \Omega\right)=\frac{1}{2} \operatorname{vol}_{n-1}\left(S_{r}(z)\right)-C_{2} \frac{r^{n}}{2} \int_{S_{z} \partial \Omega}\left\langle L_{z} \xi, \xi\right\rangle d \xi+O\left(r^{n+1}\right) \\
& =\frac{\operatorname{vol}_{n-1}\left(S^{n-1}\right)}{2} r^{n-1}-C_{2} \frac{\operatorname{vol}_{n-2}\left(S^{n-2}\right)}{2} H_{\partial \Omega}(z) r^{n}+O\left(r^{n+1}\right),
\end{aligned}
$$

using $H_{\partial \Omega}(z)=\operatorname{tr}\left(L_{z}\right) /(n-1)$. This finishes the proof of the proposition.

Proof of Proposition 1.19, Introducing

$$
B_{1}(r):=\operatorname{vol}_{n}\left(B_{r}(x) \cap \Omega\right) \text { and } B_{2}(r)=\operatorname{vol}_{n}\left(B_{r}(\pi(x)) \cap \mathcal{S}(\Omega)\right) \text {, }
$$

we first note that

$$
B_{1}(r) \leq B_{2}(r) \text { for all } r \geq 0 .
$$

Namely, properties (S1) and (S3) imply that

$$
\mathcal{S}\left(B_{r}(x) \cap \Omega\right) \subset B_{r}(\pi(x)) \cap \mathcal{S}(\Omega)
$$

and we obtain (11) immediately with the help of property (S2). Moreover, we have $B_{j}(0)=0$ and there is a constant $r_{0}>0$ such that

$$
B_{1}\left(r_{0}\right)=B_{2}\left(r_{0}\right)=\operatorname{vol}_{n}(\Omega) .
$$

Let $g_{M}:[0, \infty) \rightarrow(0, \infty)$ be the strictly decreasing function satisfying $k_{M}(x, y)=$ $g_{M}(d(x, y))$ for all $x, y \in M$ (see property (HK3)). Inequality (5) follows now from the following integration by parts argument for Stiltjes integrals:

$$
\begin{aligned}
\int_{\Omega} k_{M}(x, y) d y & =\int_{0}^{r_{0}} g_{M}(r) d B_{1}(r) \\
& =g\left(r_{0}\right) B_{1}\left(r_{0}\right)+\int_{0}^{r_{0}} B_{1}(r) d\left(-g_{M}(r)\right) \\
& \leq g\left(r_{0}\right) B_{2}\left(r_{0}\right)+\int_{0}^{r_{0}} B_{2}(r) d\left(-g_{M}(r)\right) \\
& =\int_{0}^{r_{0}} g_{M}(r) d B_{2}(r)=\int_{\mathcal{S}(\Omega)} k_{M}(\pi(x), y) d y .
\end{aligned}
$$

The proof of strict inequality in the non-symmetric case needs some harder work. W.l.o.g, we can assume that $x=\pi(x)$ and $E_{x}=E$. From the above arguments it 
suffices to prove that there is a non-empty open interval $I$ such that $B_{1}(r)<B_{2}(r)$ for all $r \in I$. $E$ bounds two closed half planes $H_{1}, H_{2} \subset M$. For $z \in E$, let $h_{z}$ denote the $g$-line through $z$ and $\lambda_{z}$ denote the Riemannian measure of the submanifold $h_{z} \subset M$. We introduce the functions $f_{j}: E \times[0, \infty) \rightarrow[0, \infty)$ by

$$
f_{j}(z, r):=\lambda_{z}\left(B_{r}(x) \cap \Omega \cap H_{j} \cap h_{z}\right) .
$$

Note that the sets $\Omega_{j}:=\Omega \cap H_{j}$ can be reconstructed from the functions $f_{j}$ up to measure zero. Thus (essential) non-symmetry of $\Omega$ means that $f_{1}$ and $f_{2}$ are different measurable functions. In particular, there exists a radius $\rho>0$ such that $f_{1}(\cdot, \rho), f_{2}(\cdot, \rho) \in L^{1}(E)$ do not coincide. Let $\lambda$ denote the Riemannian measure of the hyperplane $E \subset M$. Assume that $f_{1}(\cdot, \rho)>f_{2}(\cdot, \rho)$ on a set of positive measure. For all $L>0,0<m<n$ we introduce the sets

$$
\begin{aligned}
A_{L, m, n}:=\left\{w \in E_{x} \mid L \leq f_{1}(w, \rho)+f_{2}(w, \rho)\right. & \leq L+1 / n \\
& \text { and } \left.f_{1}(w, \rho) \geq f_{2}(w, \rho)+1 / m\right\} .
\end{aligned}
$$

Our assumption implies that we can find a point $z \in E$ and $L>0,0<m<n$ appropriately such that we have, for every open neighborhood $U \subset E$ of $z$ :

$$
\lambda\left(U \cap A_{L, m, n}\right)>0 .
$$

For any small number $\epsilon>0$ (to be specified later), we can choose an open interval $I$ and a neighborhood $U$ of $z$ such that we have, for all $r \in I$ and $w \in U$ :

$$
L-\epsilon<\lambda_{w}\left(B_{r}(x) \cap h_{w}\right)<L .
$$

Then we have, for all $w \in U \cap A_{L, m, n}$ and all $r \in I$,

$$
\lambda_{w}\left(B_{r}(x) \cap \mathcal{S}(\Omega) \cap h_{w}\right)=\lambda_{w}\left(B_{r}(x) \cap h_{w}\right) .
$$

Since $f_{2}(w, \rho) \leq \frac{L}{2}+\frac{1}{2 n}-\frac{1}{2 m}$ we obtain, on the other hand,

$$
\begin{aligned}
\lambda_{w}\left(B_{r}(x) \cap \Omega \cap h_{w}\right) & =f_{1}(w, r)+f_{2}(w, r) \leq \frac{1}{2} \lambda_{w}\left(B_{r}(x) \cap h_{w}\right)+f_{2}(w, \rho) \\
& <\lambda_{w}\left(B_{r}(x) \cap h_{w}\right)+\left(\frac{1}{2 n}+\frac{1}{2} \epsilon\right)-\frac{1}{2 m} .
\end{aligned}
$$

Since $m<n$ we can choose $\epsilon>0$ originally small enough such that

$$
\lambda_{w}\left(B_{r}(x) \cap \Omega \cap h_{w}\right)<\lambda_{w}\left(B_{r}(x) \cap \mathcal{S}(\Omega) \cap h_{w}\right)-\delta,
$$

for a suitable small $\delta>0$, for all $r \in I$, on a set of positive measure in $U$. Since we have, for all other $w \in E$ :

$$
\lambda_{w}\left(B_{r}(x) \cap \Omega \cap h_{w}\right) \leq \lambda_{w}\left(B_{r}(x) \cap \mathcal{S}(\Omega) \cap h_{w}\right),
$$

we conclude with Fubini that $B_{1}(r)<B_{2}(r)$ for all $r \in I$. This finishes the proof of strict inequality in the non-symmetric case.

\section{Appendix A. A square-Shaped DumbBell}

Consider the initial temperature distribution $\chi_{\Omega}$ where $\Omega$ consists of two squares of the same side length $a>0$ connected by a rectangle with the side lengths $b, c>0$ (see Figure 8). One easily concludes that the set $H(t)$ is contained in the horizontal 


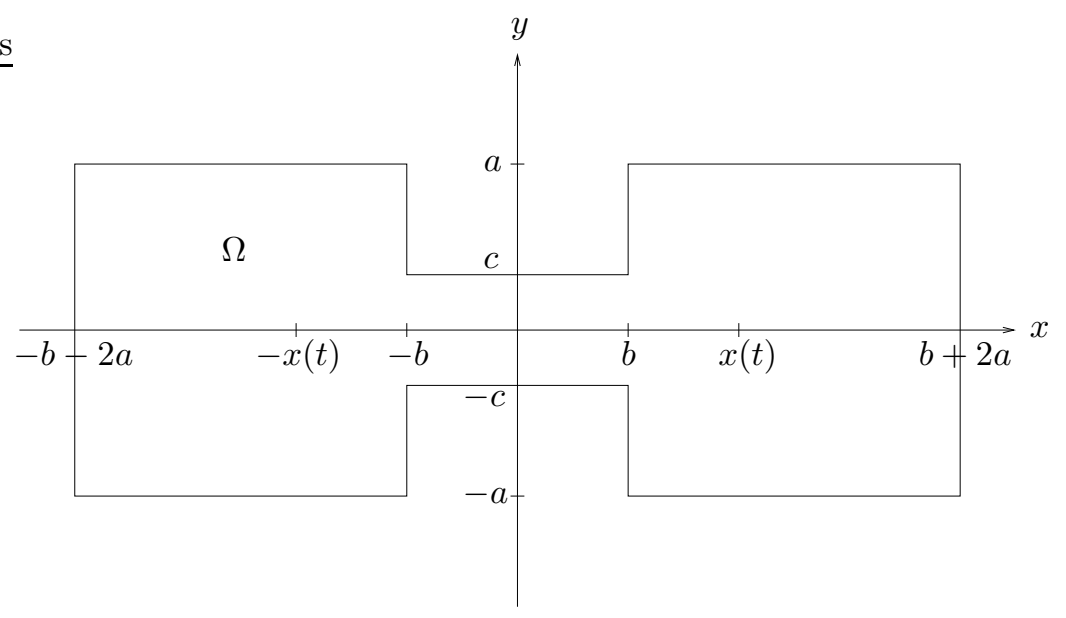

Figure 8. Movement of hottest points in $\Omega$

$x$-axis. The temperature distribution $f(t, x)$ of a point $z=(x, 0)$ at time $t>0$ is given by

$$
\begin{aligned}
2 \pi t f(t, x) & =\int_{0}^{c} e^{-y^{2} /(4 t)} d y \int_{0}^{b+2 a} e^{-\left(x-x^{\prime}\right)^{2} /(4 t)}+e^{-\left(x+x^{\prime}\right)^{2} /(4 t)} d x^{\prime} \\
& +\int_{c}^{a} e^{-y^{2} /(4 t)} d y \int_{b}^{b+2 a} e^{-\left(x-x^{\prime}\right)^{2} /(4 t)}+e^{-\left(x+x^{\prime}\right)^{2} /(4 t)} d x^{\prime} .
\end{aligned}
$$

For fixed $t>0$, we have

$$
\begin{aligned}
& \pi e^{x^{2} /(4 t)} \frac{\partial f}{\partial x}(t, x)=\left(\int_{c}^{a} e^{y^{2} /(4 t)} d y\right) e^{-b^{2} /(4 t)} \sinh \left(\frac{b}{2 t} x\right) \\
& \quad-\left(\int_{0}^{a} e^{y^{2} /(4 t)} d y\right) e^{-(b+2 a)^{2} /(4 t)} \sinh \left(\frac{(b+2 a)}{2 t} x\right) .
\end{aligned}
$$

Introducing

$$
h_{t}(x):=\frac{\sinh \left(\frac{b}{2 t} x\right)}{\sinh \left(\frac{b+2 a}{2 t} x\right)},
$$

the extrema of $f(t, \cdot)$ are at $x=0$ and at the $x$-solutions of

$$
h_{t}(x)=\frac{\int_{0}^{a} e^{-y^{2} /(4 t)} d y}{\int_{c}^{a} e^{-y^{2} /(4 t)} d y} e^{-\left(a^{2}+a b\right) / t} .
$$

For fixed $t>0$, the function $h_{t}$ is even and strictly decreasing to zero on $[0, \infty)$ with $h_{t}(0)=b /(b+2 a)$. Consequently, the condition (12) has precisely one solution $x(t)$ in $(0, \infty)$ iff

$$
g(t):=\frac{\int_{0}^{a} e^{-y^{2} /(4 t)} d y}{\int_{c}^{a} e^{-y^{2} /(4 t)} d y} e^{-\left(a^{2}+a b\right) / t}<\frac{b}{b+2 a},
$$

and no solution, otherwise. Moreover, in the first case we can conclude that $x(t) \in$ $(0, b+a)$, because for $x_{1}=b+a$ we obtain

$$
h_{t}\left(x_{1}\right)<e^{\frac{b}{2 t} x_{1}-\frac{b+2 a}{2 t} x_{1}}=e^{-\left(a^{2}+a b\right) / t} \leq g(t),
$$


where we used the estimate

$$
\frac{\sinh u}{\sinh v}<e^{u-v} \quad \text { for all } 0 \leq u<v
$$

Note that

$$
\lim _{t \rightarrow 0} g(t)=0, \quad \lim _{t \rightarrow \infty} g(t)=\frac{a}{a-c}>\frac{b}{b+2 a},
$$

and that $g:[0, \infty) \rightarrow[0, \infty)$ is a product

$$
g(t)=\frac{1}{\int_{c}^{a} e^{\left(4 a^{2}-y^{2}\right) /(4 t)} d y} \cdot \int_{0}^{a} e^{-y^{2} /(4 t)} d y \cdot e^{-a b / t}
$$

of three strictly increasing continuous functions. Therefore there is a unique $t_{0}>0$ such that

$$
g\left(t_{0}\right)=\frac{\int_{0}^{a} e^{-y^{2} /\left(4 t_{0}\right)} d y}{\int_{c}^{a} e^{-y^{2} /\left(4 t_{0}\right)} d y} e^{-\left(a^{2}+a b\right) / t_{0}}=\frac{b}{b+2 a} .
$$

We conclude that the set $H(t)$ of hottest points at time $t$ is given by $\{-x(t), x(t)\} \subset$ $(-b-a, b+a)$ for $0<t<t_{0}$ and that both temperature maxima collapse at the origin at time $t=t_{0}$. For $t>t_{0}$, the origin is the only temperature maximum. Equation (13) allows to calculate the critical time $t_{0}$ up to any precision. In the case $c=0$ we obtain

$$
t_{0}=\frac{1}{4} \frac{(b+2 a)^{2}-b^{2}}{\ln (b+2 a)-\ln (b)} .
$$

For fixed $a, b>0$ the time of collapse $t_{0}=t_{0}(a, b, c)$ becomes arbitrarily small as c $\nearrow a$.

\section{Appendix B. A tube about a space Curve}

Let $C \subset \mathbb{R}^{3}$ be a smooth space curve, $c:[a, b] \rightarrow \mathbb{R}^{3}$ be an arc length parametrization of $C$,

$$
\begin{aligned}
f_{1}(t) & :=\dot{c}(t), \\
f_{2}(t) & :=\frac{\ddot{c}(t)}{\|\ddot{c}(t)\|}, \\
f_{3}(t) & :=f_{1}(t) \times f_{2}(t)
\end{aligned}
$$

be the accompagnying Frenet trihedron and $\kappa, \tau$ the curvature and the torsion of $c$. Let $0<R<\frac{1}{\max \kappa(t)}$ and $\Sigma$ be the closed tube of radius $R$ about $C$.

We fix $t_{0} \in[a, b]$ and set $\kappa_{0}:=\kappa\left(t_{0}\right)$. For every angle $\alpha \in[0,2 \pi)$ we introduce the plane

$$
E_{\alpha}=\mathbb{R} g_{1}(\alpha)+\mathbb{R} g_{2}(\alpha)
$$

with

$$
g_{1}(\alpha)=f_{1}\left(t_{0}\right) \quad \text { and } g_{2}(\alpha)=\cos \alpha f_{2}\left(t_{0}\right)+\sin \alpha f_{3}\left(t_{0}\right) .
$$

There is a canonical identification of $E_{\alpha}$ with $\mathbb{R}^{2}$ via $(x, y) \mapsto x g_{1}(\alpha)+y g_{2}(\alpha)$.

Let $z_{ \pm}:=c\left(t_{0}\right) \pm R g_{2}\left(t_{0}\right) \in \partial \Sigma$. The intersection $\partial \Sigma \cap\left(c\left(t_{0}\right)+E_{\alpha}\right)$ consists, locally near $z_{-}$and $z_{+}$, of two plane curves. Let $C_{\alpha} \subset c\left(t_{0}\right)+E_{\alpha}$ denote the component of this intersection through $z_{+}$. We call $C_{\alpha}$ the distance $R$ curve of $C$ in direction $\alpha$ (see Figure 9). We first prove the following result: 


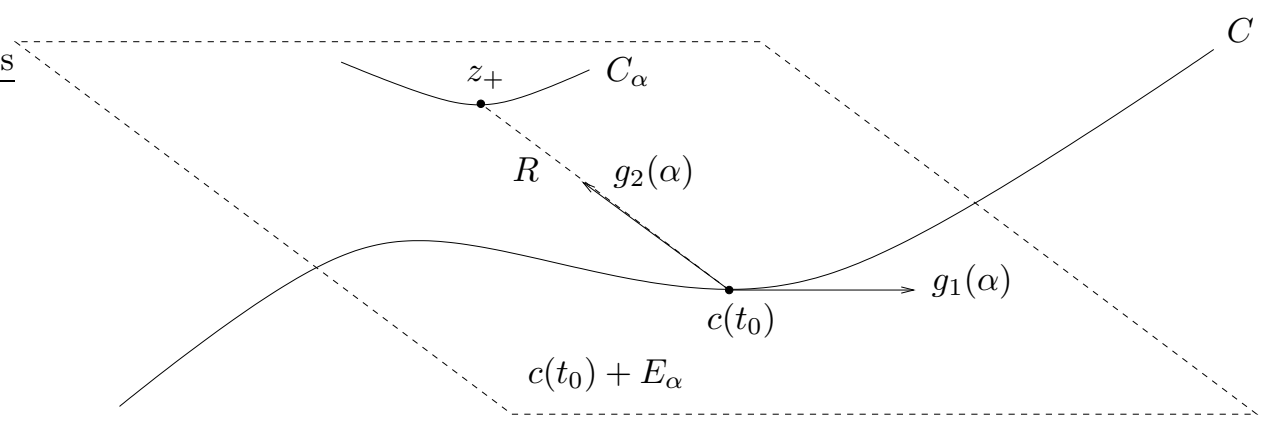

Figure 9 . The distance $R$ curve $C_{\alpha}$ of $C$ in direction $\alpha$

Proposition B.1. Let $C_{\alpha}$ be the distance $R$ curve of $C$ in direction $\alpha \in[0,2 \pi)$ and $c_{\alpha}:\left(t_{0}-\epsilon, t_{0}+\epsilon\right) \rightarrow \mathbb{R}^{3}$ be a parametrization of $C_{\alpha}$ near $z_{+}=c\left(t_{0}\right)+R g_{2}\left(t_{0}\right)$ satisfying

$$
\left|c(t)-c_{\alpha}(t)\right|=R \quad \text { for all } t \in\left(t_{0}-\epsilon, t_{0}+\epsilon\right) .
$$

Let $\kappa_{0} \geq 0$ denote the curvature of $C$ in $c\left(t_{0}\right)$. Then we have

$$
\begin{aligned}
& \dot{c}_{\alpha}\left(t_{0}\right)=\left(1-R \kappa_{0} \cos \alpha\right) g_{1}(\alpha), \\
& \ddot{c}_{\alpha}\left(t_{0}\right)=C_{1} g_{1}(\alpha)+\kappa_{0} \cos \alpha\left(1-R \kappa_{0} \cos \alpha\right) g_{2}(\alpha) .
\end{aligned}
$$

(The constant $C_{1}$ is of no importance for our considerations.)

Proof. Let $\alpha \in[0,2 \pi)$ be fixed. Let $x, y:\left(t_{0}-\epsilon, t_{0}+\epsilon\right) \rightarrow \mathbb{R}$ denote the coordinate functions of the curve $c_{\alpha}$ in the plane $c\left(t_{0}\right)+E_{\alpha}$, i.e.,

$$
\begin{aligned}
c_{\alpha}(t) & =c\left(t_{0}\right)+x(t) g_{1}(\alpha)+y(t) g_{2}(\alpha) \\
& =c\left(t_{0}\right)+x(t) f_{1}\left(t_{0}\right)+y(t)\left(\cos \alpha f_{2}\left(t_{0}\right)+\sin \alpha f_{3}\left(t_{0}\right)\right) .
\end{aligned}
$$

Note that $x(0)=0$ and $y(0)=R$.

(14) implies that there is a smooth function $\alpha:\left(t_{0}-\epsilon, t_{0}+\epsilon\right) \rightarrow \mathbb{R}$ with $\alpha(0)=\alpha$ and

$$
c_{\alpha}(t)=c(t)+R\left(\cos \alpha(t) f_{2}(t)+\sin \alpha(t) f_{3}(t)\right) .
$$

Using (15), (16) and the Frenet equations, we conclude that

$$
\begin{aligned}
& \dot{c}_{\alpha}(t)=\dot{x}(t) f_{1}\left(t_{0}\right)+\dot{y}(t)\left(\cos \alpha f_{2}\left(t_{0}\right)+\sin \alpha f_{3}\left(t_{0}\right)\right) \\
= & (1-R \kappa(t) \cos \alpha(t)) f_{1}(t)+R(\dot{\alpha}(t)+\tau(t))\left(-\sin \alpha(t) f_{2}(t)+\cos \alpha(t) f_{3}(t)\right) .
\end{aligned}
$$

Comparison of coefficients at $t=t_{0}$ implies that

$$
\dot{\alpha}\left(t_{0}\right)+\tau\left(t_{0}\right)=0,
$$

i.e., we have

$$
\dot{c}_{\alpha}\left(t_{0}\right)=\left(1-R \kappa_{0} \cos \alpha\right) g_{1}(\alpha) .
$$

Differentiating again and using again the Frenet equations, we obtain

$$
\begin{aligned}
\ddot{c}_{\alpha}(t)= & \ddot{x}(t) f_{1}\left(t_{0}\right)+\ddot{y}(t)\left(\cos \alpha f_{2}\left(t_{0}\right)+\sin \alpha f_{3}\left(t_{0}\right)\right) \\
= & (-R \dot{\kappa}(t) \cos \alpha(t)+R \kappa(t) \dot{\alpha}(t) \sin \alpha(t)) f_{1}(t) \\
& +\left(\kappa(t)-R \kappa^{2}(t) \cos \alpha(t)-R(\ddot{\alpha}(t)+\dot{\tau}(t)) \sin \alpha(t)\right) f_{2}(t) \\
& +R(\ddot{\alpha}(t)+\dot{\tau}(t)) \cos \alpha(t) f_{3}(t)+(\dot{\alpha}(t)+\tau(t)) v(t),
\end{aligned}
$$


with a suitable vector valued function $v:\left(t_{0}-\epsilon, t_{0}+\epsilon\right) \rightarrow \mathbb{R}^{3}$. Using $\dot{\alpha}\left(t_{0}\right)+\tau\left(t_{0}\right)=$ 0 , the comparison of the coefficients of $f_{2}$ and $f_{3}$ at $t=t_{0}$ yields

$$
R\left(\ddot{\alpha}\left(t_{0}\right)+\dot{\tau}\left(t_{0}\right)\right)=\kappa_{0} \sin \alpha\left(1-R \kappa_{0} \cos \alpha\right) .
$$

Inserting this back into (17) we end up with

$$
\begin{aligned}
\ddot{c}_{\alpha}\left(t_{0}\right)=C_{1} f_{1}\left(t_{0}\right)+ & \left(1-R \kappa_{0} \cos \alpha\right)\left(\kappa_{0}-\kappa_{0} \sin ^{2} \alpha\right) f_{2}\left(t_{0}\right) \\
& +\left(1-R \kappa_{0} \cos \alpha\right) \kappa_{0} \sin \alpha \cos \alpha f_{3}\left(t_{0}\right) \\
& =C_{1} g_{1}(\alpha)+\left(1-R \kappa_{0} \cos \alpha\right) \kappa_{0} \cos \alpha g_{2}(\alpha) .
\end{aligned}
$$

This finishes the proof of the proposition.

Next we calculate the planar curvature $\hat{\kappa}_{\alpha}$ of $C_{\alpha} \subset c\left(t_{0}\right)+E_{\alpha}$ in $z_{+}$. Let

$$
g_{3}(\alpha)=g_{1}(\alpha) \times g_{2}(\alpha)=-\sin \alpha f_{2}+\cos \alpha f_{3} \perp E_{\alpha}
$$

and

$$
J: E_{\alpha} \rightarrow E_{\alpha}, \quad J(v)=g_{3}(\alpha) \times v
$$

be rotation in $E_{\alpha}$ by $\pi / 2$. Then Proposition B.1 implies that the planar curvature of $c_{\alpha}$ in $t_{0}$ is given by

$$
\hat{\kappa}_{\alpha}=\frac{\left\langle\ddot{c}_{\alpha}\left(t_{0}\right), J\left(\dot{c}_{\alpha}\left(t_{0}\right)\right)\right\rangle}{\left\|\dot{c}_{\alpha}\left(t_{0}\right)\right\|^{3}}=\frac{\kappa_{0} \cos \alpha}{1-R \kappa_{0} \cos \alpha} .
$$

We are now able to calculate the asymptotics of

$$
\epsilon \mapsto A(R+\epsilon)=\operatorname{vol}_{2}\left(S_{R+\epsilon}\left(c\left(t_{0}\right)\right) \cap \Sigma\right) .
$$

Using Fermi coordinates on the sphere $S_{R+\epsilon}\left(c\left(t_{0}\right)\right)$ about the great circle

$$
S_{R+\epsilon}\left(c\left(t_{0}\right)\right) \cap\left(c\left(t_{0}\right)+\mathbb{R} g_{2}(\alpha)+\mathbb{R} g_{3}(\alpha)\right)
$$

we obtain

$$
\begin{aligned}
A(R+\epsilon) & =4 \pi(R+\epsilon)^{2}-(R+\epsilon)^{2} \int_{0}^{2 \pi} \int_{-\theta_{\alpha}(\epsilon)}^{\theta_{\alpha}(\epsilon)} \cos \theta d \theta d \alpha \\
& =4 \pi(R+\epsilon)^{2}-2(R+\epsilon)^{2} \int_{0}^{\pi}\left(\sin \theta_{\alpha}(\epsilon)+\sin \theta_{\alpha+\pi}(\epsilon)\right) d \alpha,
\end{aligned}
$$

where the angle $\theta_{\alpha}(\epsilon) \in[0, \pi / 2]$ is described in Figure 10 (Note that in Figure 10, $S_{R+\epsilon}\left(c\left(t_{0}\right)\right)$ denotes the circle about $c\left(t_{0}\right)$ of radius $R+\epsilon$ in the plane $c\left(t_{0}\right)+E_{\alpha}$ and not the 2-dimensional sphere.) Applying Proposition 1.9 (with curvature $\kappa=$ $-\hat{\kappa}_{\alpha}=-\kappa_{0} \cos \alpha /\left(1-R \kappa_{0} \cos \alpha\right)$, seen from $\left.c\left(t_{0}\right)\right)$ we conclude that

$$
\sin \theta_{\alpha}(\epsilon) \sim \theta_{\alpha}(\epsilon)=\frac{\sqrt{2 R}}{R+\epsilon}\left(\sqrt{1-R \kappa_{0} \cos \alpha}+o(1)\right) \epsilon^{1 / 2} .
$$

Consequently, we obtain

$$
\begin{aligned}
& A(R+\epsilon)=4 \pi(R+\epsilon)^{2} \\
& -\sqrt{8 R}(R+\epsilon)\left(\int_{0}^{\pi} \sqrt{1-R \kappa_{0} \cos \alpha}+\sqrt{1+R \kappa_{0} \cos \alpha} d \alpha+o(1)\right) \epsilon^{1 / 2},
\end{aligned}
$$

which implies that

$$
0 \leq \kappa\left(t_{1}\right)<\kappa\left(t_{2}\right) \Longrightarrow\left(c\left(t_{2}\right), \Sigma, \mathbb{R}^{3}\right) \succ\left(c\left(t_{1}\right), \Sigma, \mathbb{R}^{3}\right),
$$

similarly as in the two-dimensional case. 


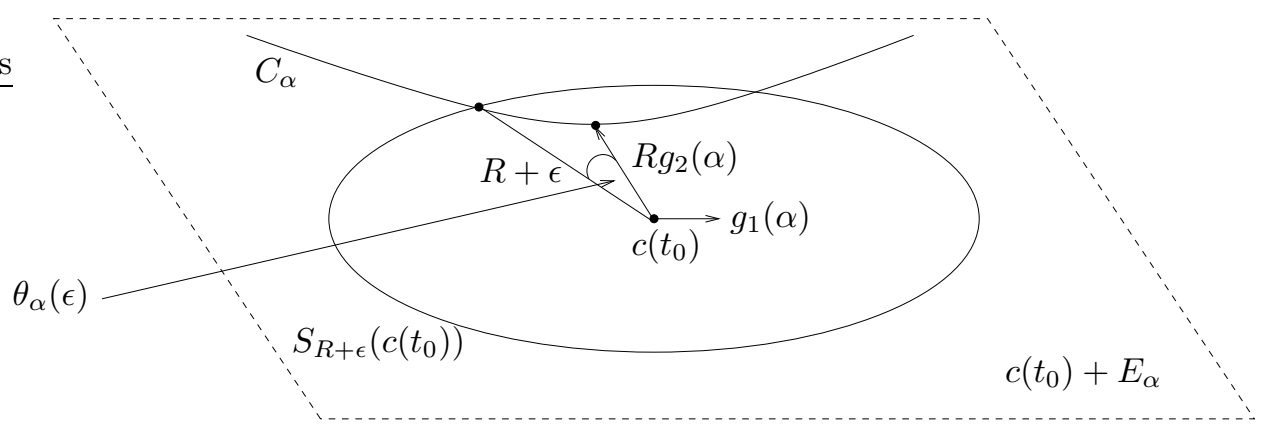

Figure 10 . The angle $\theta_{\alpha}(\epsilon)$

\section{Appendix C. Small time asymptotics of the heat KeRnel}

The aim of this appendix is to prove Corollary C.1 below, a result, which was used in earlier sections. Let $M$ be a complete Riemannian manifold with lower bound $-\kappa<0$ on the Ricci curvature, upper bound $K>0$ on the sectional curvature and lower positive bound $i_{0}$ on the injectivity radius. For any open subset $U \subset M$ let $k_{U}^{D}(t, x, y)$ denote the corresponding Dirichlet heat kernel.

Theorem 4 (Principle of not feeling the boundary). Let $I \subset M$ be a compact subset and $U \subset M$ be an open subset containing $I$ and $\epsilon>0$. Then there exists a constant $C>0$, depending only on $\operatorname{dim} M, \kappa, K$, and $i_{0}$, such that we have for all $x, y \in I$ and all $t \in\left(0, \sqrt{i_{0} / 2}\right]:$

$$
\left|k_{M}(t, x, y)-k_{U}^{D}(t, x, y)\right| \leq C e^{-d^{2} /(4+\epsilon) t},
$$

where $d \in(0, \infty]$ denotes the distance between $I$ and $\partial U$.

Proof. Let $x, y \in I$ and $t \in\left(0, \sqrt{i_{0} / 2}\right]$ be fixed and, for small $\delta>0$, let $f_{\delta} \in C^{\infty}(M)$ be a non-negative function with support in $B_{\delta}(y) \subset U$ and total integral one. Let

$$
g(t, x)=\int_{U} k_{M}(t, x, z) f_{\delta}(z) d z \quad \text { and } g^{D}(t, x)=\int_{U} k_{U}^{D}(t, x, z) f_{\delta}(z) d z .
$$

Since $k_{M} \geq k_{U}^{D}$, we conclude that

$$
h=g-g^{D}:[0, \infty) \times \bar{U} \rightarrow[0, \infty)
$$

is a solution of the heat equation with $h(0, x)=0$ for all $x \in \bar{U}$ and $h(s, x) \geq 0$ for all $(s, x) \in(0, \infty) \times \partial U$. The maximum principle (see, e.g., [Tay-96, Section 6.1]) implies that

$$
h(t, x) \leq \max _{(s, w) \in(0, t] \times \partial U} h(s, w) .
$$

For every $(s, w) \in(0, t] \times \partial U$ we have with [LY-86, Cor.3.1]

$$
\begin{array}{r}
0 \leq h(s, w)=\int_{U}\left(k_{M}(s, w, z)-k_{U}^{D}(s, w, z)\right) f_{\delta}(z) d z \leq \int_{B_{\delta}(y)} k_{M}(s, w, z) f_{\delta}(z) d z \\
\leq \max _{z \in B_{\delta}(y)} k_{M}(s, w, z) \leq C \exp \left(\frac{-(d-\delta)^{2}}{(4+\epsilon) t}\right)
\end{array}
$$


since $y \in I, w \in \partial U$ and $d\left(B_{\delta}(y), \partial U\right) \geq d-\delta$. Note that $C>0$ depends only on the parameters mentioned in the theorem. Letting $\delta \rightarrow 0$ we finally derive from (18) that

$$
0 \leq k_{M}(t, x, y)-k_{U}^{D}(t, x, y) \leq C \exp \left(\frac{-d^{2}}{(4+\epsilon) t}\right),
$$

finishing the proof of the theorem.

Corollary C.1. Let $I \subset M$ be a compact subset and $0<\delta<\min _{x \in I} \operatorname{inj}(x)$. Then we have, for all $x \in I$ and $y \in B_{\delta}(x)$ :

$$
k_{M}(t, x, y)=\frac{1}{(4 \pi t)^{n / 2}} e^{-d^{2}(x, y) /(4 t)}\left(u_{0}(x, y)+O(t)\right),
$$

with a uniform $O(t)$ and $u_{0}$ given by the Minakshisundaram-Pleijel expansion.

Proof. We choose an open set $U \subset M$ with $d(I, \partial U)>0$ large enough. The Minakshisundaram-Pleijel expansion for the Dirichlet heat kernel $k_{U}^{D}$ yields, for all $x \in I$ and $y \in B_{\delta}(x)$ :

$$
k_{U}^{D}(t, x, y)=\frac{1}{(4 \pi t)^{n / 2}} e^{-d^{2}(x, y) /(4 t)}\left(u_{0}(x, y)+O(t)\right) .
$$

We conclude with Theorem 4 that the same asymptotics holds true for the heat kernel $k_{M}$.

\section{REFERENCES}

[ALT-91] A. Alvino, P.L. Lions and G. Trombetti. Comparison results for elliptic and parabolic equations via symmetrization: a new approach, Differential and Integral Equations 4 (1991), $25-50$.

[BGM-72] M. Berger, P. Gauduchon, and E. Mazet. Le Spectre d'une Variété Riemannienne, Lecture Notes Math. 194, Springer-Verlag, New York, 1971.

[Cha-84] I. Chavel. Eigenvalues in Riemannian Geometry, Academic Press, New York, 1984.

[ChK-90] I. Chavel and L. Karp. Movement of hot spots in Riemannian manifolds, Journal d'Analyse Math. 55 (1990), 271-286.

[FeT-73] G. Fejes Tóth. Sum of moments of convex polygons, Acta Math. Acad. Sci. Hungar. 24, 417-421.

[Flo-93] A. Florian. Extremum problems for convex discs and polyhedra, Handbook of convex geometry - Vol. A (ed. P.M. Gruber and J.M. Wills, North-Holland, Amsterdam, 1993), 177221.

[Gri-99] A. Grigor'yan. Estimates on heat kernels on Riemannian manifolds, in Spectral Theory and Geometry (B. Davies and Y. Safarov, eds.), Cambridge University Press, Cambridge, 1999, $140-225$.

[Gue-03] F. Guéritaud. A note on Steiner symmetrization of hyperbolic triangles, Elem. Math. 58 (2003), 21-25.

[KP-02] L. Karp and N. Peyerimhoff. Extremal properties of the principal Dirichlet eigenvalue for regular polygons in the hyperbolic plane, Arch. Math. 79 (2002), 223-231.

[LY-86] P. Li and S.-T. Yau. On the parabolic kernel of the Schrödinger operator, Acta Math. 156 (1986), 153-201.

[Pey-02] N. Peyerimhoff. Simplices of maximal volume or minimal total edge length in hyperbolic space, J. London Math. Soc. 66 (2002), 753-768.

[SchY-94] R. Schoen and S.-T. Yau. Lectures on Differential Geometry, International Press Incorporated, Boston, 1994.

[Tay-96] M.E. Taylor. Partial Differential Equations, Springer-Verlag, New York, 1996.

LEON KARP

Department of Mathematics, CUNY Graduate Center and Lehman College

365 Fifth Avenue, New York, NY 10016, USA 
email: lkarp@gc.cuny.edu

Norbert PEyerimhofF

Department of Mathematical Sciences, Science Laboratories

South Road, Durham, DH1 3LE, United Kingdom

email: norbert.peyerimhoff@durham.ac.uk 